\title{
TRANSMISSION OF THIN LIGHT BEAMS \\ THROUGH \\ TURBULENT MIXING LAYERS
}

\author{
John B. Wissler " and Anatol Roshko** \\ Graduate Aeronautica1 Laboratories \\ California Institute of Technology \\ Pasadena, California 91125
}

\begin{abstract}
This research investigates the effects of spanwise and streamwise coherent structures in a turbulent mixing layer on the deflection of a thin light beam which is transmitting transyersely through the mixing layer from the high-speed side to the low speed side. Both equal and unequal density mixing layers of varying pressures and velocities are studied, using a lateral effect detector to dynamically track the motion of a $\mathrm{He}-\mathrm{Ne}$ laser beam. Beam deflections in the streamwise direction are found to be associ ated mainly with the spanwise coherent structures; at low Reynolds Numbers the beam deflection is directly related to the part of a spanwise structure through which the beam passes. Maximum deflections are associated with the trailing edge of the spanwise coherent structures. Spanwise deffections are caused mainly by the streamwise coherent structures and as such exhibit large variations across the span of the flow. With the development of the streamwise structures, spanwise deflections are found to exceed streamwise deflections. Mixing transition, as scaled using the momentum thickness of the high-speed side, is found to cause a peak in the rms fluctuations of both the streamwise and spanwise deflections.
\end{abstract}

\section{NOMENCLATURE}

d light beam diameter

p static pressure

$S R \quad$ Strehl Ratio

$t$ time

$U$ free stream velocity

$x$ downstream distance

y spanwise distance

$z \quad$ light path direction of transmission

B. Gladstone-Dale constant

$\delta$ mixing layer thickness, turbulent region thickness

* Captain, USAF, now at Phillps Laboratory, Kirtand AFB, Albuquerque, NM; Mernber AIAA.

** Theodore von Kámin Professor of Aeronantics; Feliow AIAA. . 
$\theta_{x} \quad$ streamwise deflection angle

$\theta_{y} \quad$ spanwise deflection angle

$\delta_{1}^{* *}$ momentum thickness on the high-speed side

$\Lambda$ turbulent eddy size

to most amplified initial wavelength

$\mu \quad$ kinematic viscosity

$P$ density

$\sigma_{\theta} \quad$ ms fluctuation of deflection angle

Subscript

$S C$ fully scaled

$S \quad$ standard conditions

$x \quad$ streamwise direction

$y$ spanwise direction

1 high-speed side

2 low-speed side

Superscript

* pressure-scaled

\section{INTRODUCTION}

Aero-optics is the study of light transmission through aerodynamic flows, where-in "density inhomogeneities in the flow-freld can perturb...the ability to propagate [light] beams through the flowfield (Sutton, 1985)." That is, the effect of inhomogeneities in a turbulent flow-field is to aberrate light beams which are passing through the it. For example, these aberrations can have adverse effects on the ability of aircraft-mounted sensors to resolve objects outside the aircraft flow-field (Sutton, 1985, and Elliot et al, 1989).

Past treatment of these aerodynamic flows for the purposes of aero-optic analysis treated the turbulence as being isotropic or homogeneous. However, it is known that turbulent shear flows (boundary layers, mixing layers, jets). contain large-scale "coherent structures", which suggests that the assumptions of isotropy or homogeneity may be too limiting.

The objective of this paper is to experimentally examine the relationship between the large-scale structure and the behavior of a thin beam of light which is being transmitted through a mixing layer, at incidence normal to its plane. The approach is to use a fast-response beam-defiection measurement device to dynamically measure the angular deflection of a thin $\mathrm{He}-\mathrm{Ne}$ laser beam which is being perturbed by the turbulent flow past it.

The paper begins with a background on those aspects of aero-optics and fluid mechanics which are relevant to the experiment, then follows with a description of the experiments. Results are presented for mixing layers between streams of different densities and also for the case of equal densities. 


\section{BACKGROUND}

The question of relative sizes of the light beam, the path length through the turbilent region, and the scale of the turbulence is important, because it facilitates the organization of the research into groups having common characteristics and it determines scaling relationships in theoretical analysis. Scale sizes are indicated by beam diameter $(d)$, path length through the turbulent region $(\delta)$, and turbulence eddy size $(\Lambda)$. Traditionally, aero-optics investigations have been characterized by assumptions and analysis based on the following relationships:

$$
\Lambda \ll \delta \ll d
$$

Those investigations have been concemed with transmitting large beams through shear layers or boundary layers. When applied to transmission through the atmosphere, the relationships become as follows:

$$
d \ll \Lambda \ll \delta
$$

In order to achieve the spatial resolution required for understanding how the flow structure affects beam transmission, this study makes use of a thin beam and the following relationship:

$$
d \ll A \sim \delta
$$

In the mixing layer, because of the presence of large-scale structures, the turbulence scale size $\Lambda$ is on the order of the transmission path length $\delta$. Thus the beam may experience only one or two large deflections in its passage through the turbulent region.

Recenty. Truman and Lee (1990) used geometrical optics to numerically study the effects of organized turbulent structures on phase distortion in optical beam transmission through a numerically simulated homogeneous turbulent shear flow. They showed that the most intense refractive index fluctuations are closely correlated with the presence of hairpin vortices and that, because of these vortices, optical beam quality is highly dependent on the angle of transmission through the shear layer.

Chew and Christiansen (1990, 1991), in an experimental study, and Tsai and Christiansen (1990), in a related numerical study, investigated the effects of large-scale stuctires in a turbulent mixing layer on the Strehl Ratio of relatively large diameter light beams transmitting through the mixing layer onhogonally to the plane of the layer. Strehl Ratio, or $S R$, is the ratio of the actual, aberrated beam intensity to the beam intensity under ideal conditions. The two studies found that, by forcing the layer and influencing the development of the large-scale structures, it was possible to improve $S R$. An additional finding in the experimental study was that mixing transition is associated with a large decrease in $S R$.

The presence of large-scale structures in the mixing layer implies that $\Lambda \sim \delta$ (Equation 3). The above noted research efforts investigated coherent structure effects on large beams of light, such that $\Lambda_{1}$ $\delta \ll d$. In order to obtain spatial resolution of the effects of the structure, in the research reported here use was made of thin beams, so that the relation in Equation (3) is applicable. This is perhaps closest to the situation studied by Liepmann (1952) in his analysis of the statistical effect of turbulence on a ray 
of light traversing a boundary layer, but at that time no connection was made with coherent structure.

The two kinds of large-scate structures of interest in the mixing layer are the spanwise coherent structures, called primary vortices, and the streamwise coherent structures; called secondary vortices. The spanwise coherent structures appear on side-view shadowgraph pictures as roller-like formations which have a characteristic appearance (Fig. 1). For the purposes of this paper, three features of this structure are important: the braid between the individual structures, the vortex core of the spanwise structures within which the high- and low-speed fluids are mixed, and what we will call the "cusp", which is visible at the trailing edge of each primary vortex. In Fig. 1 the plan view shows streamwise oriented streaks that mark the edges of the secondary vortices, which occur in counter-rotating pairs (Bemal, 1981). They develop to full strength somewhat later than the spanwise structures.

Together, these two kinds of structure have a major influence on how the fluids from the respective streams are stirred together and mixed. In the course of its development from laminar initial conditions, the flow progresses from being basically two-dimensional and dominated by the spanwise coherent structures to being a three-dimensional network of both primary and secondary vortices. The progression from two- to three-dimensional flow causes a large increase in the extent to which the fluids from each side are mixed together in the mixing layer. This increase in the "mixedness" of the flow is called "mixing transition" and has been observed in both gases and liquids (Konrad, 1976; Breidenthal. 1978; and Dimotakis and Koochesfahani, 1986). It implies a large increase of the interfacial area between the two fluids. On spark shadowgraphs, the transition appears to occur suddenly, as marked by an increase in small-scale structure, but defined from time-averaged concentration measurements the mixing transition extends over a finite distance because the streamwise location of the instantaneous mixing transition front varies with time.

Because of the change in the character of the flow as it progresses through mixing transition, the optical quality of the mixing layer changes also. For this reason, it is useful to know where mixing transition occurs. The mixing transition was at first correlated with the large-scale Reynolds number $\operatorname{Re}_{\delta}$ (Konrad, 1976), where $\delta(x)$ is the mixing-layer thickness, and a nominal value $\operatorname{Re}_{\delta}=10^{4}$ was used. This corresponds to approximately the end of the transition, which actually extends over a finite region. For a given flow $\mathrm{Re}_{\delta}$ increases, approximately linearly, with downstream distance $x$. An alternative and more convenient parameter is the nondimensional downstream distance $x / \delta_{1}^{* *}$, where $\delta_{1}^{* *}$ is the initial momentum thickness. Using this as the goveming parameter, mixing transition extends over values of $x / \delta_{1}^{* *}$ from about 150 to 1000 (BreidenthaI, 1978; Bemat, 1981; Roshko, 1991).

\section{THEORY}

The problem of the transmission of thin light beams through turbulent mixing layers may be treated using ray optics, refraction being the primary effect of interest. The optical parameter which is measured in the experiment is the tilt or deflection of a thin light beam. The incoming wavefronts are assumed to be initially planar and the rays are assumed to be undeviated. Because the flow is incompressible, the only source of optical abberation is from the mixing of the two dissimilar gases (c.g. $\mathrm{He}$ and $\mathrm{N}_{2}$ ). As such, the refractive index inside the mixing layer may be expressed as a function of the concentration of the slow-speed fluid $c_{2}$ where $c_{2}=c_{2}(x, y, z, t)$. 


$$
n=1+\frac{\mathrm{p}}{\mathrm{P}_{s}} \frac{T_{s}}{T}\left[\beta_{1}+c_{2}\left(\beta_{2}-\beta_{1}\right)\right]
$$

Using geometrical optics (e.g. Liepmann and Roshko, 1957), the bending angte of a single ray of light which has passed through the flow-field may be expressed as follows.

$$
\begin{gathered}
\theta_{i}=K_{1} \int_{L_{1}(x, y \neq)}^{L_{2}(x y, t)} \frac{\partial c_{2} / \partial x_{i}}{K_{2}+K_{1} c_{2}} d z \\
K_{1}=\frac{\mathrm{p}}{\mathrm{p}_{s}} \frac{T_{s}}{T}\left(\beta_{2}-\beta_{1}\right) \\
K_{2}=1+\frac{\mathrm{p}}{\mathrm{p}_{s}} \frac{T_{s}}{T} \beta_{1}
\end{gathered}
$$

In Equation (5), $i=1$ or 2 (i.e., $x$ or $y$ ) and coordinates are as shown in Figure 1. Therefore, the deflection angle is dependent only on the instantaneous value of $c_{2}$ distribution over the light path at each location in the mixing layer. Equation (5) may be simplified by considering that $K_{1} \ll 1, K_{2} \doteq 1$, and $0<c_{2}<1$ ( $\beta$, the Gladstone-Dale constant of the fluid, is of order $10^{-5}$ ). The result is shown in Equation (6) for $\theta_{x}$ only; the expression is similar for $\theta_{y}$.

$$
\frac{1}{K_{1}} \theta_{x} \doteq \int_{L_{1}}^{L_{2}} \frac{\partial c_{2}}{\partial x} d z
$$

From the above relations, the importance of a nonzero difference in Gladstone-Dale constant across the mixing layer, the existence of nonzero concentration gradients, and the existence of a path length over which the gradients can work is apparent. Given that the intermixed fluids have different GladstoneDale constants, as they are entrained from the freestreams by the vortical structures, interfaces are formed between regions of relatively homogeneous fluid mixture (see also Dimotakis and Koochesfahani, 1986). Because of diffusive effects, the interfaces have finite thickness, nevertheless smaller than the experimental light beam diameter. As the light beam travels through the mixing layer, it is deflected according to Equation (6) at the interfaces between uniform regions; within a uniform region it travels in a straight line as determined by the interfaces previously encountered. The kind of interface encountered by the light is dependent on the location of the light with respect to the coherent structure.

Equation (6) also shows that the results of different experiments can be scaled by the pressures at which the experiments are run and by the Gladstone-Dale constant shift across the layer, since the temperature varies Iittle between experiments. For this reason, all the results of the experiments are first divided by the ratio $\mathrm{p} / \mathrm{p}$, so that any angle $\theta$ now becomes a "pressure-scaled" $\theta^{*}$ (with an $x$ or $y$ subscript to denote a streamwise or spanwise deflection angle). This allows comparison between experiments of the same gas combination. A second scaling, accounting for the different gases used, is referred to as "fully-scaled" and denoted by $\theta_{S C}$ with the appropriate $x$ or $y$ subscript. The scaling is summarized in the following equations: 


$$
\begin{gathered}
-6- \\
\theta^{*}-\frac{\theta}{p / p_{s}} \\
\theta_{S C}-\frac{\theta^{*}}{\Delta \beta}
\end{gathered}
$$

\section{APPARATUS}

The experiments were done in the apparatus originally used by Brownand Roshko (1974) (also Konrad, 1976; Bemal, 1981). It provides a vertically-flowing mixing layer enclosed inside a tark that is rated to 8 bars. The mixing layer-is fed by two banks of eight high pressure gas bottles and is controlled by needle valves and upstream and downstream (back) pressure regulators. Optical data are acquired through two glass windows and electronic data are acquired through electrical feedthroughs. Two optical tables of equal height straddle the facility; these have steel tops for use with magnetic bases and are joined by aluminum I-beams for rigidity. In Fig. 2, which shows a view of the facility, the flow in the test section is into the page.

Data were acquired with an Everex System 1800 microcomputer equipped with an RC Electronics, Inc̈. IS-16E Computerscope data acquisition system (1 MHz total sample rate). The laser beam deflection was measured by a UDT Sensors, Inc. SC-10D dual-axis lateral effect detector. The detector generates eurrents proportional to the beam's displacement along each axis; these currents are amplified and converted to voltages by two UDT Instruments, Inc. Model 301DIV 30kHž amplifiers, one per axis. The voltages were-dynamically recorded by the microcomputer. The angular deflection is obtained from the ratio of the displacement and the distance between the mixing layer and the detector.

Experiments were nun at pressures of 2, 4, and 6 bar with velocity ratios of 0.38 for all experiments. Several values of velocity, less than or equal to $10 \mathrm{~m} / \mathrm{s}$, and two gas combinations were used. A density ratio of $1 / 7$ was achieved using $H e$ as the high-speed gas and $N_{2}$ tas the low-speed gas. For a density ratio of unity, $\mathrm{N}_{2}$ was used as the high-speed gas and a mixture of 32 percent $\mathrm{He}$ and 68 percent $A r$, by mole fraction;-as the low-speed gas. The experimental conditions are listed in Täbles 1 and 2.

\section{RESULTS}

The results are discusssed by first considering the effects of the spanwise coherent structures, then the effects of the streamwise coherent structures, and finally the effects of mixing transition. Within each discussion the results for both the unequal density and equal density experiments will be covered.

It is important to note that the overall refractive index shift across the mixing layer, as determined by $\Delta \beta\left({ }_{3} \beta_{2}-\beta_{1}\right)$, controls the polarity of the beam deffection trace. Since the unequal and equal density mixing layers, as they were set up in the flow facility, have $\Delta \beta$ 's of opposite sign, the resulting deflection traces are of opposite sign. 


\section{Effects of Spanwise Coherent Structures}

As shown in Fig. I the instantaneous structure of the mixing layer consists of a series of spanwise coherent structures which are convecting downstream past the fixed laser beam, and causing the beam to deflect in a regular, characteristic manner. By using hot-wire data and spark-generated shadowgraph and light sheet data (Wissler, 1991), it is possible to relate different portions of the signal waveform to characteristic parts of the spanwise coherent structure. These features are especially apparent at low Reynolds numbers.

Figure 3 shows a typical portion of the time trace of the pressure-scaled $\theta_{x}^{*}$, at a low Reynolds number. Also shown is the simultaneous $\theta_{y}^{*}$ trace. Because of the convective nature of the spanwise coherent structures, the laser beam passes through, in successive and repetitive order, a cusp, a braid, and a core (see Fig. 1). The most visible and striking feature of the trace is the large-amplitude, negative-topositive swing in $\theta_{x}^{*}$. For the coordinate system used, and with $N_{2}$ and $\mathrm{He}$, these large negative swings correspond to upstream deflections in the laser beam (e.g., ' $A$ ' in Fig. 3). This portion of the signal' is attributable to the region of the spanwise coherent structure just downstream of the cusp. The upstream (i.e., negative-valued) deflection is immediately followed by a positive or downstream deflection of the laser beam which is associated with the braid between spanwise coherent structures (e.g. 'B' in Fig. 3). Thus, the passage of the cusp corresponds to the negative-to-positive swing in the defiection angle (' $A$ ' to ' $B$ ' in Fig. 3). As the braid convects past the beam, the angle begins a swing back in the negative direction (' $\mathrm{B}$ ' to ' $\mathrm{C}$ ' in Fig. 3). Eventually the leading edge of the core of the next spanwise coherent structure crosses the beam axis, which produces a momentary hesitation or shoulder in the trace (' $\mathrm{C}$ ' in Fig. 3). At this point the bean is inside the core and the deflection angle progresses toward the next negative extremum (' $D$ ' in Fig. 3) at which time the process repeats itself.

Occasionally, the structure of the mixing layer breaks down, i.e., the large-scale structure loses its coherency, with a corresponding effect on the beam deflection signal, as is also shown in Fig. 3 at $t \sim 0.31 \mathrm{~s}$. After a short time span the instabilities reestablish themselves and the regular signal reappears (e.g., at $t \sim 0.34 \mathrm{~s}$ ).

The effect of increasing the Reynolds number was investigated by increasing the tank pressure. increasing the flow velocity, and/or positioning the laser beam farther downstream from the splitter plate. Figure 4 shows a typical example of the effect on both $\theta_{ \pm}^{*}$ and $\theta_{y}^{*}$ of increasing the Reynolds number. The most noticeable effect on $\theta_{x}^{*}$ is an increase in the small-scale, random component of the signal. As the Reynolds number is further increased, this component can grow to the point where it tends to obscure the underlying beam signature (Fig. 4). Frequently, as in Fig. 4 for $t \doteq 0.30 s$ and $0.34 s$, the canonical signature reappears after being obscured by the random component $(t<0.29 s)$. The location of mixing transition undergoes temporal variations in the streamwise direction; the reappearence of the canonical signal is possibly due to a downstream shift in the transition. As the Reynolds number is increased further, the canonical $\theta_{x}^{*}$ deflections become further obscured by the increased random component (Fig. 5).

While the effects of increasing Reynolds number are apparent for $\theta_{x}^{*}$, they are more dramatic for the $\theta_{y}^{*}$ signature. As shown by the progression in Reynolds number in Figs. 3 to 5 , which were taken at the 
same location, there is a large increase in the magnitude of the $\theta_{y}^{*}$ signal, particularly in the positive peaks which are coincident with the negative peaks of the $\theta_{x}^{*}$. signals, implying that the positive peaks occur simultaneously with the passing of the cusp by the laser beam. At the highest values of Reynolds number, the peaks in $\theta_{y}^{*}$. exhibit a tendency to be of either sign, and there are more small-scale fluctuations in the signal.

The equal-density mixing layer $\left(N_{2} / H e-A r\right)$ generates beam deflection signatures very similar to those of the unequal density mixing layer. Figure 6 shows a typical low-Reynolds-number trace in which the structural influences are labelled as in Fig. 3. (For convenient comparison, Fig. 6 is shown on the same page as Fig. 3. and, because $\Delta \beta<0$, the trace has been printed with reversed polarity.) The magnitude of $\Delta \beta$ is about one third that of the unequal density case, so the pressure-scaled deflection magnitudes are correspondingly reduced.

\section{Effects of Streamwise Coherent Structures}

The streamwise coherent structures have a large effect on the deflection of the laser beam. This effect is most apparent in spanwise profiles of the r.m.s. values, $\sigma_{\theta_{x}}$ and $\sigma_{\theta_{j}}$; measured at 1 to $2 \mathrm{~mm}$ intervals. As will be shown, the variations in $\sigma_{\theta_{j} ;}$ across the span, are larger than the variations in $\sigma_{\theta_{x}^{*}}$. As expected, the streamwise vortices, display most of their effects in the spanwise deflections of the laser beam.

Figures $7 \mathrm{a}-\mathrm{c}$ show $\sigma_{\theta_{x}^{*}}$ and $\sigma_{\theta_{j}^{*}}$ plotted as function of spanwise position (2 mm spacing for $10 \mathrm{~mm}$ either side of an arbitrary reference position near the centerline of the test section; the test section is actually $100 \mathrm{~mm}$ wide) for three different streamwise positions $(x=45,88$, and $113 \mathrm{~mm})$. At $x=45 \mathrm{~mm}, \sigma_{\theta_{*}}$. shows negligible variation across the span of the flow. At the middle downstream position, the spanwise variations in $\sigma_{\theta_{x}^{*}}$ are greater, approximately $25 \mu \mathrm{rad}$, while at the farthest downstream position the variations are slightly less.

When attention is tumed to the ms fluctuations of the spanwise deflection angle $\sigma_{\theta} ;$, much greater variation with spanwise position is evident. At the farthest upstream position, the variation is approximately $150 \mu \mathrm{rad}$. This increases to approximately $175 \mu \mathrm{rad}$ at the middle position $(x=88 \mathrm{~mm})$ and then decreases to approximately $125 \mu \mathrm{rad}$ at the farthest downstream position. It is interesting that, once the streamwise structures develop, they cause similar spanwise variations in both $\sigma_{\theta_{i}^{*}}$ and $\sigma_{\theta_{i}^{*}}$, which implies that there is some cross-coupling between the orthogonal sets of structures.

Figures 8a-f show temporal histories of the streamwise and spanwise pressure-scaled beam deflections for six different spanwise positions spaced $2 \mathrm{~mm}$ apart at $x=45 \mathrm{~mm}$. As one would expect from Fig. $7 a$, the $\theta_{x}^{*}$ signal shows the canonical waveform for all six positions. The $\theta_{y}^{*}$ trace is much more sensitive to spanwise position and does not display any characteristic signature common to each position. (Note that the secondary structures are not convecting spanwise.) However, common to each $\theta_{y}^{*}$ trace is a positive or negative peak in deflection angle which is coincident with the passage of the cusp past the laser beam.

In the case of the equal density mixing layer, spanwise profiles of the ms fluctuation (Figs. 9a-b) show 
less spanwise variation than the unequal density mixing layer, although the general pattem of the variations is the same. The traverses were made at $1 \mathrm{~mm}$ increments for a total of 5 spanwise positions. In general, the spanwise variations in $\sigma_{\theta_{y}^{*}}$ are coupled with the changes in $\sigma_{\theta_{x}^{*}}$.

The correlations between different runs of the equal density mixing layer at a given spanwise position are not as consistent as with the unequal density mixing layer. This may be attributed to the fact that the spanwise scales of the equal density mixing layer are smaller (recall that $\delta_{1}^{* *}$ for the equal density mixing layer is smaller than for an equiyalent condition in the unequal density mixing layer). At small downstream distances, the spanwise flow structure is much finer, which limits the resolution available with the $1 \mathrm{~mm}$ laser beams traversing in $1 \mathrm{~mm}$ increments. At large downstream distances, even though the spacing of streamwise structures has become larger, the flow is in a post-transition state, those. structures are no longer confined to preferred spanwise locations, and the spanwise variation of mean values is smoothed out (Bemal and Roshko, 1986).

\section{Effects of Mixing Transition}

As indicated in Section II., the development of the mixing layer may be related to the nondimensional downstream distance $x / \delta_{1}^{* *}$. The data for both the unequal and equal density cases will be examined together using the fully-scaled ms fluctuations of the streamwise and spanwise deflection angles $\left(\sigma_{\theta_{x s c}}\right.$ and $\left.\sigma_{\theta_{x c c}}\right)$.

The initial momentum thickness on the high-speed side $\delta_{1}^{* *}$ is calculated from estimates of the most amplified wavelength $\left(\lambda_{0}\right)$, as measured on shadowgraph pictures, and the relation $\lambda_{0}=30 \delta_{1}^{* *}$. The momentum thickness is then scaled between experiments according to the relationship,

$$
\delta_{1}^{* *} \propto\left(\frac{\mu}{p U_{1}}\right)^{1 / 2}
$$

The dependence of the ms fluctuations on $x / \delta_{1}^{* *}$ is shown in Figs. $10 \mathrm{a}$ (for $\sigma_{\theta_{2 x}}$ ) and $10 \mathrm{~b}$ (for $\sigma_{\theta_{y s c}}$ ) for both the unequal and equal density cases. What appears to be a large amount of scatter in the data at each $x / \delta_{1}^{* *}$ is actually the spanwise variation due to the formation and development of the streamwise coherent structures through the mixing transition (Figs. 7 and 9). For $150<x / \delta_{1}^{* *}<1000$, the variation is the greatest. For large values of $x / \delta_{1}^{* *}$, the spanwise variations have become smaller, especially for $x / \delta_{1}^{* *}>1000$. Note that, because the initial momentum thickness $\delta_{1}^{* *}$ for the $N_{2} / H e-A r$ mixing layer is smaller than for the $\mathrm{He} / \mathrm{N}_{2}$ mixing layer, for a given physical distance downstream of the splitter plate the nondimensional distance is larger. Considering the variety of velocity and pressure levels, downstream distances, gas combinations and, especially, considering the intrinsic spanwise variation through the mixing transition, the collapse of the data in the fully scaled presentations of Fig. 10 seems satisfactory.

The changes undergone by $\sigma_{\mathrm{b} x \mathrm{sc}}$ with changing $x / \delta_{1}^{* *}$ (Fig. 10b) are much more impressive than for $\sigma_{\theta_{\Delta c}}$ (Fig. 10a), although the general trends are similar. For very low values of $x / \delta_{1}^{* *}(<200) \sigma_{\theta x c}$ is negligible compared to $\sigma_{\beta_{x x}}$, presumably because the streamwise vortices have not formed. However, as $x / \delta_{1}^{* *}$ is increased past 200 we see a rapid increase in $\sigma_{B_{30}}$, to the point where it peaks at a value of 
1.2 at $x / \delta_{1}^{* *} \doteq 400$. The spread in $\sigma_{\beta_{s c}}$ is much greater than in $\sigma_{\theta_{x \sigma}}$, with spanwise variations of approximately 0.5 to 1.2 . For $x / \delta_{1}^{* *}>400$ the magnitude and the spanwise variation of both are decreasing, and by $x / \delta_{1}^{* *} \doteq 1000$ the magnitudes have levelled off to approximately constant values: $\sigma_{\theta_{\Delta x}} \doteq 0.6$ and $\sigma_{Q_{x s c}} \doteq 0.8$.

\section{DISCUSSION}

The results presented in Section V cover both unequal $\left(\rho_{2} / \rho_{1}=7\right)$ and equal-density mixing layers at a variety of flow conditions $(x, p, u)$. Particularly under pre-transition conditions, there exists a characteristic temporal signature that repeats as each successive spanwise structure convects by the fixed laser beam. This signal suggests an asymmetric, structurally-based effect of the flow-field on light beams passing through it.

Because the time-scale of the light beam is so much shorter than the convection time-scale of the flow, to the light beam the flow appears frozen. Therefore, the light encounters a relatively-orderef, nonisotropic, and nonhomogeneous refractive index field as determined by the locations of the streamwise and spanwise coherent structures at that instant (cf. the plan-view shadowgraph in Fig. 1).

How the light beam reacts to the spanwise coherent structures is suggested by the model shown in Fig. 11. The model is two-dimensional, and does not account for the effects of the streamwise structures. (See also Figs. 1 and 3.). The pre-transition model signature contains the shoulder associated with the leading edge of the core. The portion associated with the braid can change its spatial extent depending on the spacing of the structures. If structures are spaced more closely together, more of the signal will possess characteristics associated with the core and cusp regions. From this perspective, if one is to think about control, it is afvantageous to increase spacing since the braids induce the smallest aberrations. Altematively, if the overall aperture size of the optical apparatus is not too large, then viewing could be accomplished through braids rather than through cores and cusps, by controlling transmission to coincide with the best phase.

The model also suggests the origin of the caustics that show up as bright spots and streaks in plan-view shadowgraph pictures (e.g., Fig. 1). Because caustics are the focussing of adjacent light rays into a point in space, which overexposes the shadowgraph film, consideration of bending angle gradients suggests that they occur where the beam deflection angle has negative gradient (in our coordinate system). Portions of a beam upstream of a point are bent downstream (positive bending) while portions downstream are bent upstream (negative bending). The caustics occur where the respective parts of the beam cross. In the mixing layer, this happens first at the cusp of each spanwise coherent structure (Fig. 11). As the Reynolds number increases, the negative gradient portions of the signal increase in number, the next one is that associated with the leađing edge (i.e., the shoulder). As the flow progresses into the post-transition regime, there is a larger number of negative gradient regions (Fig. 11 - "post-transition" curve). This is reflected in the growth in the number of caustics and smaller-scale structures in the shadowgraphs. It should also be noted that, by extension, positively-sloped regions imply de-focussing. As the light spreads into other parts of the flow-field, the intensity in that region decreases; on shadowgraphs the film becomes underexposed. 
Considering the effect of mixing transition on the ms fluctuations for the two kinds of mixing layers studied, it is obvious that there exists a close relationship between the onset of mixing transition and the optical aberrations induced by the flow-field. Mixing transition occurs at values of $x / \delta_{1}^{* *}$ between 150 and 1000 , beyond which the flow has relaxed into "fully developed turbulence" (Bradshaw, 1966; Roshko, 1991). The peak in ms fluctuations occurs at $x / \delta_{1}^{* *} \sim 400$, which places it near the middle of mixing transition. The large ms values in this region result mainly from single large excursions of the deffection angle (e.g. Figs. 3 and 4), implying single encounters with vortex structures. Bemal's pictures of the streamwise vortices, and those from the numerically simulated mixing layers of Moser and Rogers (1991), suggest that the streamwise vortices become especially well formed in this region; furthermore they occur at preferred spanwise locations (which accounts for the spanwise variation of mean values). Both effects would contribute to large deflection, at particular locations. But, to produce a spanwise time-varying deflection of the beam, such a vortex must be bent away from the streamwise direction; such bending does occur where the braids joiri the main rollers, i.e, in the vicinity of what we have called the "cusp". The largest excursions in $\theta_{y}$ seem to be linked to those in $\theta_{x}$ (e.g., Fig. 8e); they probably occur at scallops visible in the plan view of Fig. 1 on the upstream edge of the roller. As in Bernal's model of the vortex topology, such a scallop may be the looped end connecting two streamwise vortices, thus forming a "hairpin".

The pictures of Konrad and of Bernal indicate that in the post-transition region the streamwise vortices are spaced further apart and are no longer at preferred locations. The rms values of deflection are now more uniformly distributed spanwise and at a level intermediate to the spanwise distributed peaks and valleys at $x / \delta_{1}^{* *}=400$.

From the preceding, it is obvious that in the optical sense as well as in the mixing sense, the flow can be divided into three regions: pre-transition, transition, and post-transition. For best optical transmission, one desires to use the pre-transition regime, because the aberrations induced by the flow are the smallest. This implies moving as far upstream in $x / \delta_{1}^{* *}$ units, as possible. A less desirable alternative is the post-transition regime. Here, despite the strong aberrations, the viewing field is spatially more homogeneous, which implies that the optical characteristics across an aperture are relatively uniform and the rms level of fluctuation is lower than in the transition region. Finally, the least desirable region for viewing is the transition region $\left(150<x / \delta_{1}^{* *}<1000\right)$. The aberrations peak in this part of the flow field at $x / \delta_{1}^{* *}-400$, there are extremely strong spanwise variations, and the optical characteristics across an aperture vary greatly, which complicates both design and signal processing.

If we consider the effect of increasing $x / \delta_{1}^{* *}$ on mixedness, on rms deflection of thin beams, and on large beam aberration, the relationship between the various quantities becomes apparent. (Fig. 12). During the mixing transition $\left(150<x / \delta_{1}^{* *}<1000\right.$ ), the mixedness of the flow increases (cf, Section $\mathrm{I}$ ) as is depicted schematically in the top graph of Fig. 12. As mixing transition develops, the rms deflections of a thin light beam increase rapidly and peak at $x / \delta_{1}^{* *}-400$, after which they decrease. As the streamwise structures form and develop, $\sigma_{\theta_{j}^{*}}$ increases faster than $\sigma_{\theta_{x}^{*}}$ and becomes the larger contributor to the flow-field induced aberrations. Chew (1991), in measurements of $S R$ for large beams (cf., Section II), noted a decrease in $S R$ through mixing transition. Replotting it as $1-S R$, which is a measure of the loss of optical quality of the flow, we see how I-SR rises through mixing transition. 
However, the use of a large light beam acts to mask the peak in ms fluctuations experienced by the thin beam.

It is of interest to consider how the fulty scaled values of $\sigma_{\theta}{ }^{\circ}$ and $\sigma_{\theta, 0}{ }^{\circ}$ might vary with further increase in $x / \delta_{1}^{* *}$ and/or Reynolds number. The values (0.6 and 0.8) indicated in Fig. 12 for $x / \delta_{1}^{* *}>1000$ are tentative. A question is whether they are asymptotic, i.e. are they invariant with increasing shear layer thickness? Two effects need to be considered: (i) whether Reynolds number dependent changes of interface thicknesses have an influence; and (ii) how the increasing number of structures encountered during transmission through the mixing layer affects the result. We believe that the former is not crucial and that the latter may be the more important effect. That is, with increasing $x / \delta_{1}^{* *}$ the cores of the large rollers contain increasing numbers of smaller vortices which were rolled up earlier (cf. the sketch in Figure 11). Their effect on a transmitting beam becomes more statistical than for the singleencounter effects in the early mixing transition. An increasing number of encounters $N$ should increase the rms deflection as $\sqrt{N}$. But it is not clear whether, in fact, the number of optically active vortices continues to increase; earlier ones might be neutralized by diffusive mixing.

Related to the preceding is the question of how a mixing layer developing from an initially turbulent boundary layer differs optically from the ones which we have studied and which have laminar initial conditions. The mixing transition is quite different in the two cases (as may be inferred from the measurements of Bradshaw, 1966). The post-transition, large coherent structure, driven by the global instabililty, is thought to be independent of initial conditions (Roshko, 1981); nevertheless, the early differences may affect the intemal structure up to large distances downstream.

To summarize, while we do not expect any further, dramatic, transitional changes in the rms values of the deflections, there may be a slow change (probably an increase) with increasing $x / \delta_{1}^{* *}$.

\section{REFERENCES}

1. Bemal, L, P. (1981) "The Coherent Structure of Turbulent Mixing Layers. I. Similarity of the Primary Vortex Structure. II. Secondary Streamwise Vortex Structure." Ph. D. Thesis, California Institute of Technology.

2. Bradshaw, P. (1966) "The Effect of Initial Conditions in the Development of a Free Shear Layer," J. Fluid Mech. 26, 225-236.

3. Breidenthal, R. E. (1978) "A Chemically Reacting, Turbutent Shear Layer." Pht. D. Thesis, Califomia Institute of Technology.

4. Brown, G. L. and Roshko, A. (1974) "On Density Effects and Large Structure in Turbulent Mixing Layers, ${ }^{1 *}$ J. Fluid Mech. 64 (4); 775-816.

5. Chew, L. and Christiansen, W. H. (1990) "Coherent Structure Effects on the Optical Performance of Plane Shear Layers," AIAA J. 29 (1), 76-80.

6. Chew, L. and Christiansen, W. H. (1991) "Experimental Investigations of Free Shear Layer Optics," AIAA Paper 91-1722. 
7. Koochesfahani, M. and Dimotakis, P. E. (1986) "Mixing and Chemical Reactions in a Turbulent Liquid Mixing Layer," J. Fluid Mech. 170, 83-112.

8. Elliot, J. L. et al. (1989) "Image Quality on the Kuiper Airbome Observatory. I. Results of the first flight series." Pub1. of the Astronomical Soc. of the Pacific 101, 737-764.

9. Konrad, J. E: (1976) "An Experimental Investigation of Mixing in Two-Dimensional Turbulent Shear Flows with Applications to Diffusion-Limited Chemical Reaction." Ph, D. Thesis, California Institute of Technology.

10. Liepmann, H. W: (1952) "Deflection and Diffusion of a Light Ray Passing Through a Boundary Layer," Report SM-14397, Douglas Aircraft.

11. Moser, R. D. and Rogers, M. M. (1991) "Mixing Transition and the Cascade to Small Scales in a Plane Mixing Layer," Physics of Fluids A 3, 1128-1134.

12. Roshko, A. (1991) "The Mixing Transition in Free Shear Flows," in The Global Geometry of Turbulence, Plenum Press, (ed, J. Jimenez).

13. Roshko, A. (1981) "The Plane Mixing Layer, flow visualization results and three-dimensional effects," in The Role of Coherent Structure in Modelling Turbulence and Mixing (ed. J. Jiminez). Lecture Notes in Physics, vol. 136, pp. 208-217. Springer.

14. Sutton, G. W. (1985) "Aero-Optical Foundations and Applications," AIAA J. 23 (10), 1525-1537.

15. Truman, C. R. and Lee, M. J. (1990) "Effects of Organized Turbulence Structure on the Phase Distortion in a Coherent Optical Beam Propagating Through a Turbulent Shear Flow," Physics of Fluids A 2 (5), 851-857.

16. Tsai, Y. P. and Christiansen, W. H. (1990) "Two-Dimensional Numerical Simulation of ShearLayer Optics," AIAA J. 28 (12), 2092-2097.

17. Wissler, John B. (1991) "Transmission of Thin Light Beams through Turbulent Mixing Layers." Ph. D. Thesis, California Institute of Technology.

\section{ACKNOWLEDGEMENT}

This research was supported by the Air Force Office of Scientific Research and Phillips Laboratory under AFOSR Grant No. AFOSR-89-0552. We wish to thank Professors Donald Coles and Bradford Sturtevant for crucial suggestions for the experimental techniques. 
Table 1

Experimental Conditions for $\left(\mathrm{He} / \mathrm{N}_{2}\right)$ Mixing Layers

$$
1 \equiv \mathrm{He}, 2 \equiv \mathrm{N}_{2}
$$

\begin{tabular}{lrrrc}
$\mathrm{p}$ (bar) & $U_{1}(\mathrm{~m} / \mathrm{s})$ & $U_{2}(\mathrm{~m} / \mathrm{s})$ & $\delta_{1}^{* *}(\mathrm{~mm})$ & $\frac{\mathrm{Re}_{x}}{x}\left[\times 10^{-6}\right)\left(\mathrm{m}^{-1}\right)$ \\
\hline 2.0 & 5.0 & 1.9 & 0.33 & 0.44 \\
2.0 & 10.0 & 3.8 & 0.23 & 0.86 \\
4.0 & 3.8 & 1.4 & 0.27 & 0.67 \\
4.0 & 5.0 & 1.9 & 0.23 & 0.86 \\
4.0 & 10.0 & 3.8 & 0.16 & 1.78 \\
6.0 & 3.8 & 1.4 & 0.22 & 1.00 \\
6.0 & 5.0 & 1.9 & 0.19 & 1.33 \\
6.0 & 10.0 & 3.8 & 0.14 & 2.68 \\
& & & & \\
Note: $\frac{\operatorname{Re}_{x}}{x}=\frac{\operatorname{Re}_{\delta}}{\delta}$ & & &
\end{tabular}

Table 2

Experimental Conditions for Equal Density $\left(\mathrm{N}_{2} / \mathrm{He}-\mathrm{Ar}\right)$ Mixing Layers

$$
1 \equiv N_{2}, 2 \equiv \mathrm{He}-\mathrm{Ar}
$$

$\mathrm{p}(\mathrm{bar}) \quad U_{1}(\mathrm{~m} / \mathrm{s}) \quad U_{2}(\mathrm{~m} / \mathrm{s}) \quad \delta_{1}^{* *}(\mathrm{~mm})$

\begin{tabular}{llll}
\hline 4.0 & 2.5 & 0.95 & 0.120 \\
4.0 & 5.0 & 1.90 & 0.083
\end{tabular}



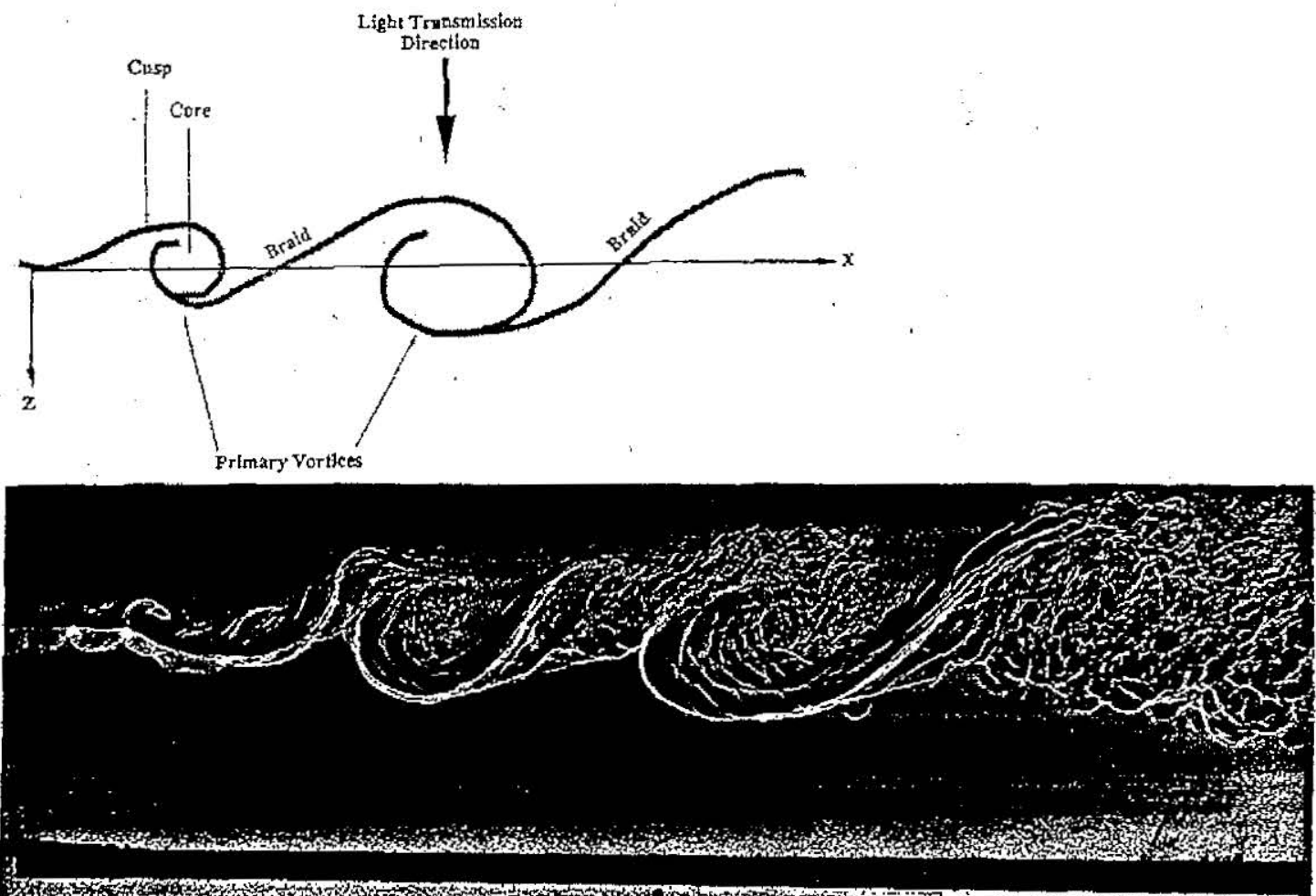

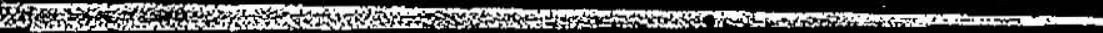

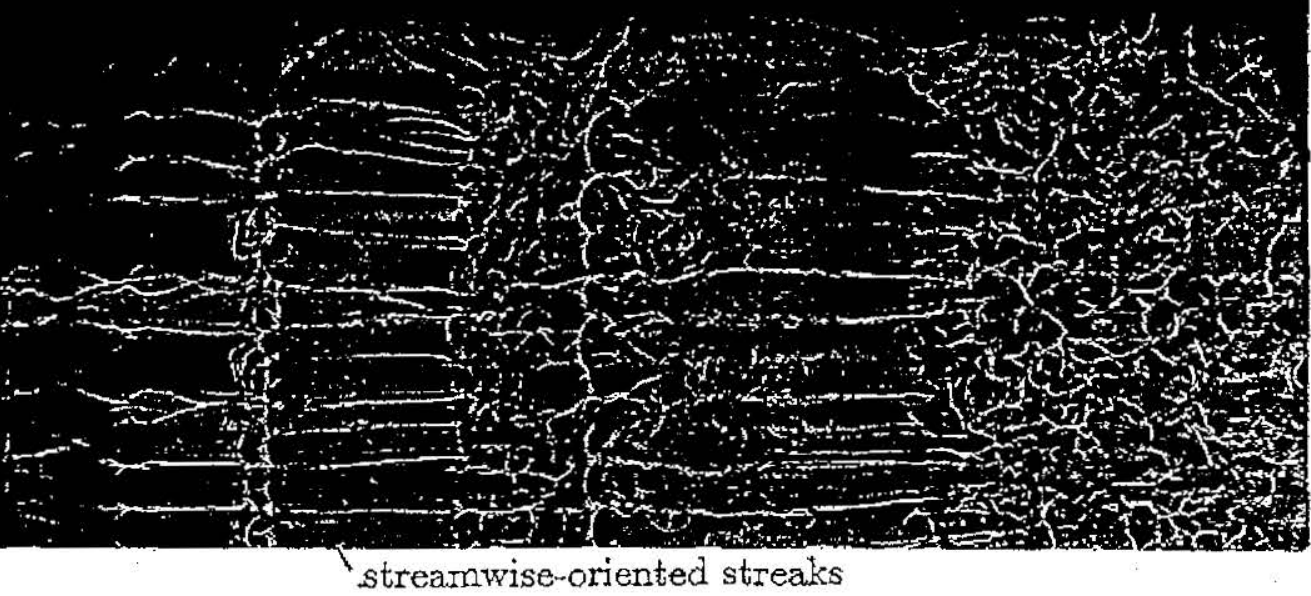

Figure 1 Views (edge and plan) of turbulent mixing layer between helium and nitrogen streams. (Definitions shown in sketch.) Shadowgraph by Konrad (1977). 


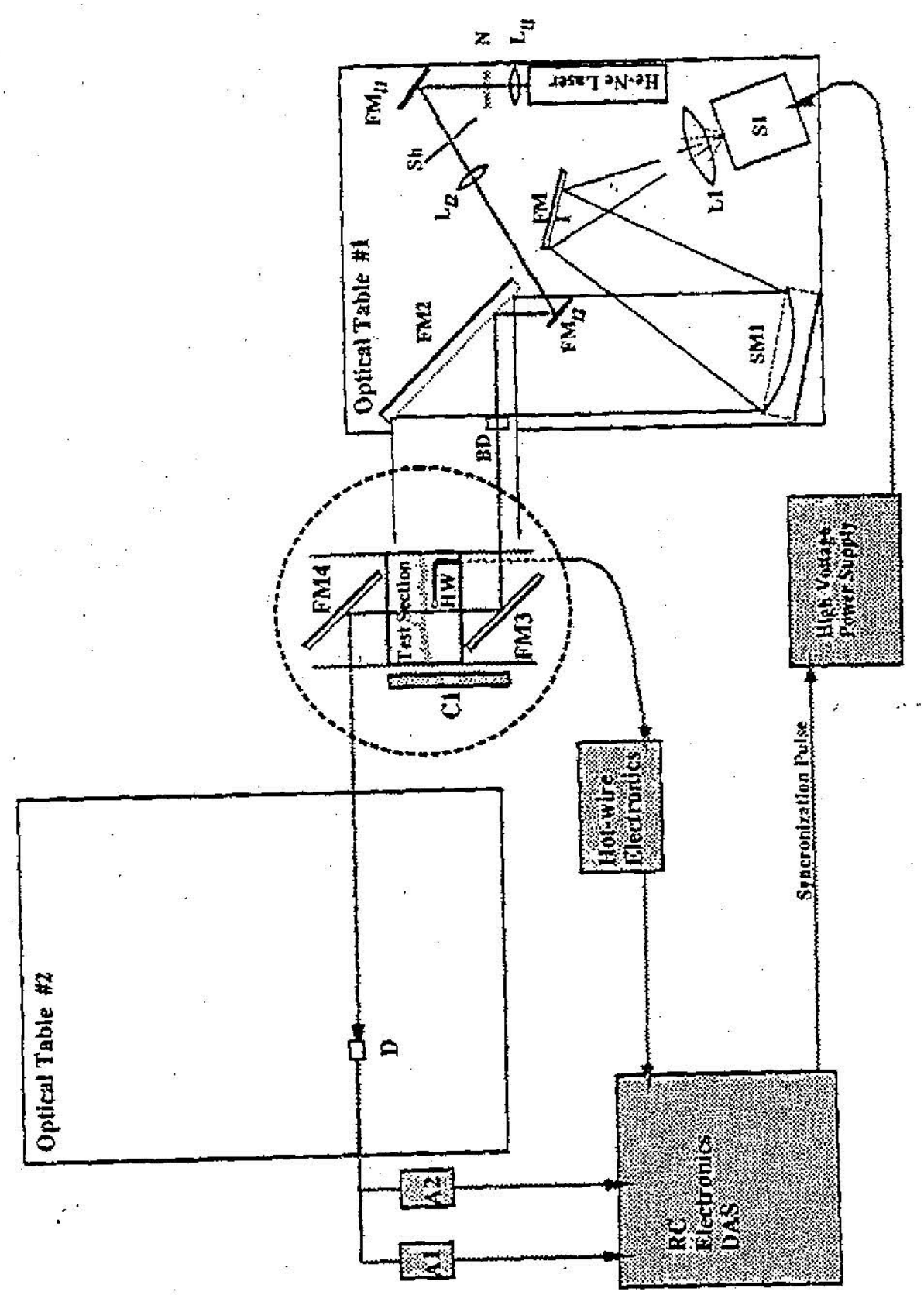

Figure 2 Experimental Set-up. 


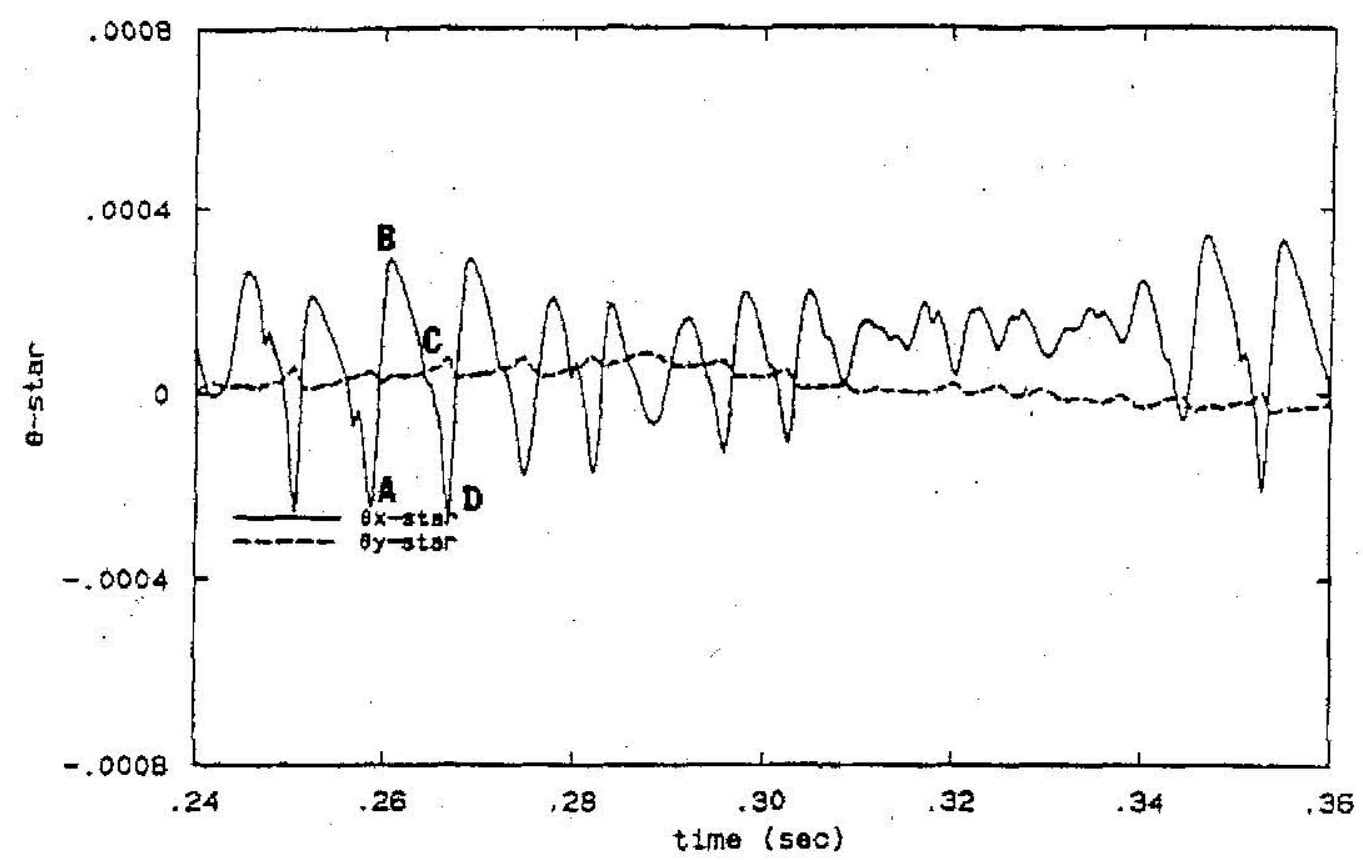

Figure 3 Pressure-scaled time traces of defiection angles. $H e-N_{2}$ mixing layer. $x=45 \mathrm{~mm}$. $\mathrm{p}=2 \mathrm{bar} ; U_{1}=5.0 \mathrm{~m} / \mathrm{s}(\mathrm{He}) ; U_{2}=1.9 \mathrm{~m} / \mathrm{s}\left(\mathrm{N}_{2}\right)$.

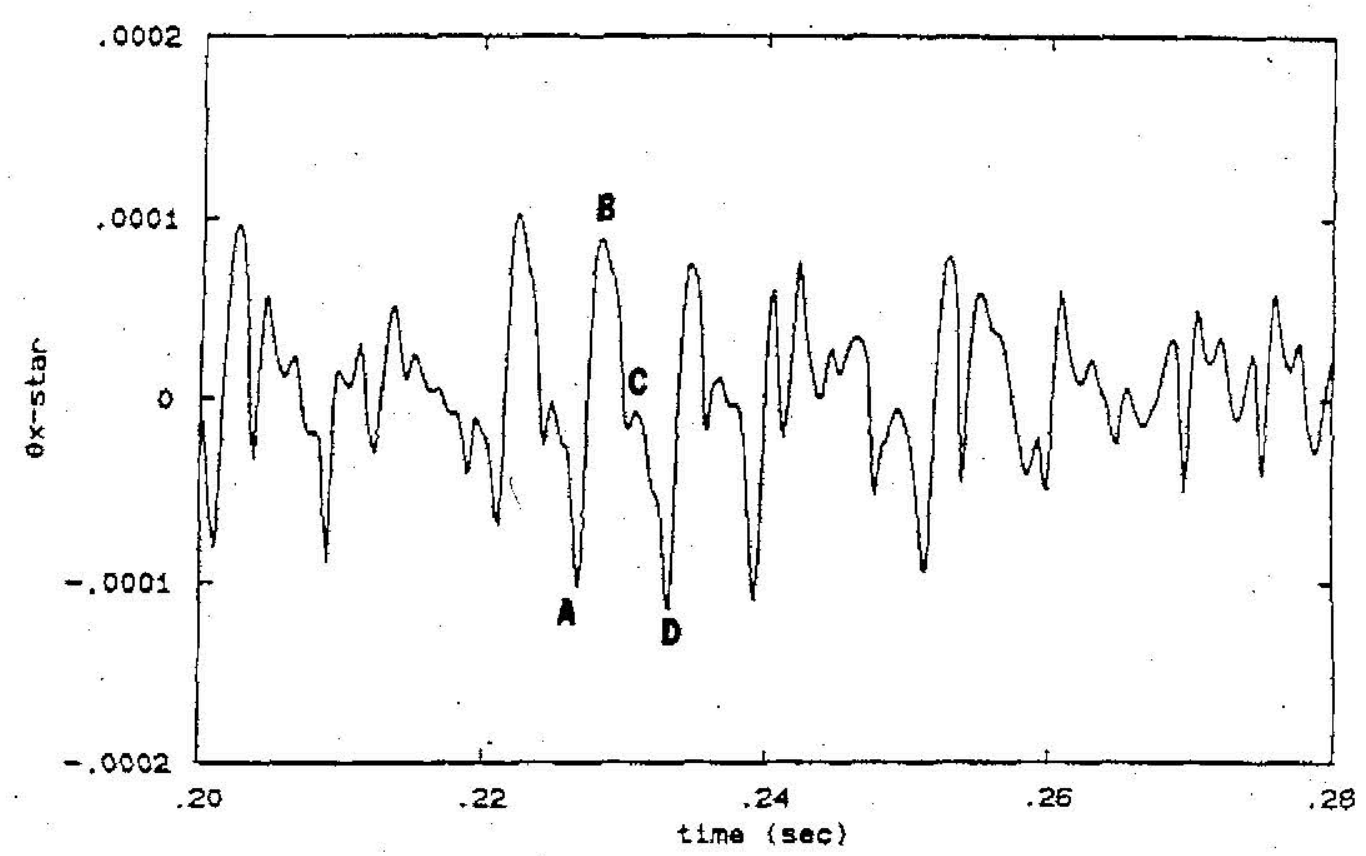

Figure 6 Pressure-scaled time traces of deflection angle $\theta_{x}^{*}$.

Uniform density $\left(N_{2} / H e-A r\right) ; p=4$ bar, $x=30 \mathrm{~mm} ; \quad U_{1}=2.5 \mathrm{~m} / \mathrm{s}$; $U_{2}=0.9 \mathrm{~m} / \mathrm{s}$. 


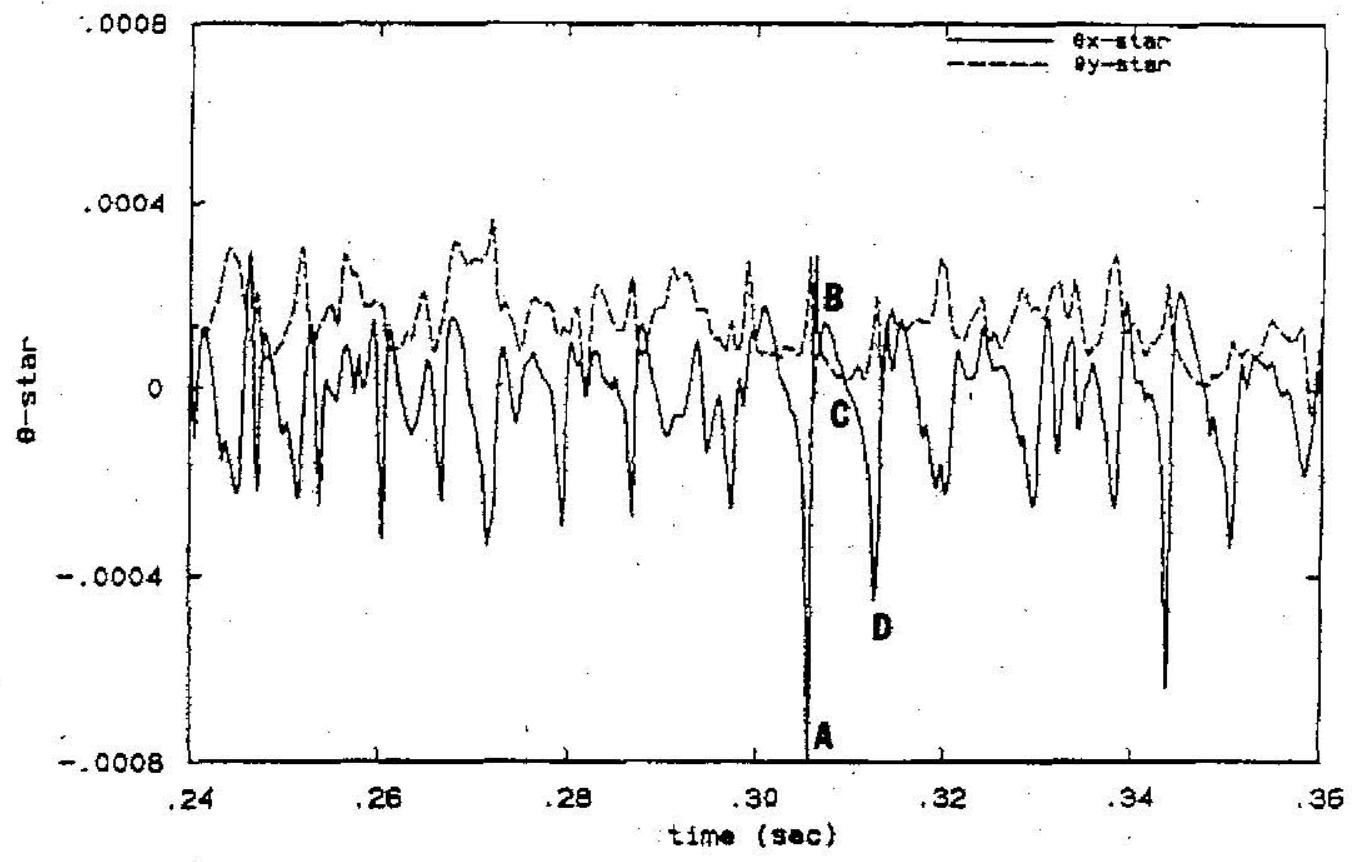

Figure 4 Pressure-scaled time traces of deflection angles. $H e-N_{2}$ mixing layer. $x=45 \mathrm{~mm}$. $\mathrm{p}=4 \mathrm{bar} ; U_{1}=5.0 \mathrm{~m} / \mathrm{s} ; U_{2}=1.9 \mathrm{~m} / \mathrm{s}$.

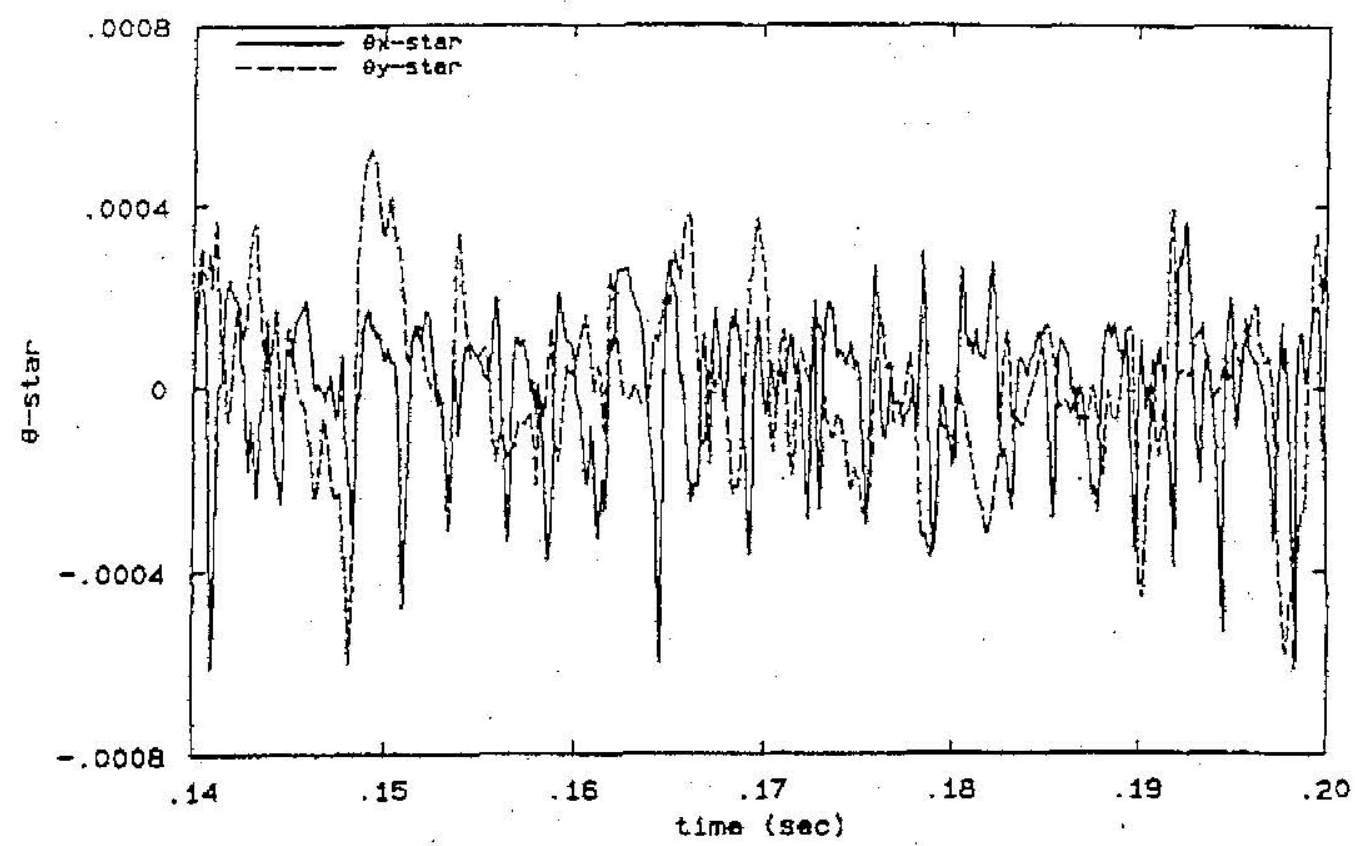

Figure 5 Pressure-scaled time traces of deflection angles. $\mathrm{He}-\mathrm{N}_{2}$ mixing layer. $x=45 \mathrm{~mm}$. $p=4 \mathrm{bar} ; U_{1}=10.0 \mathrm{~m} / \mathrm{s} ; U_{2}=3.8 \mathrm{~m} / \mathrm{s}$. 


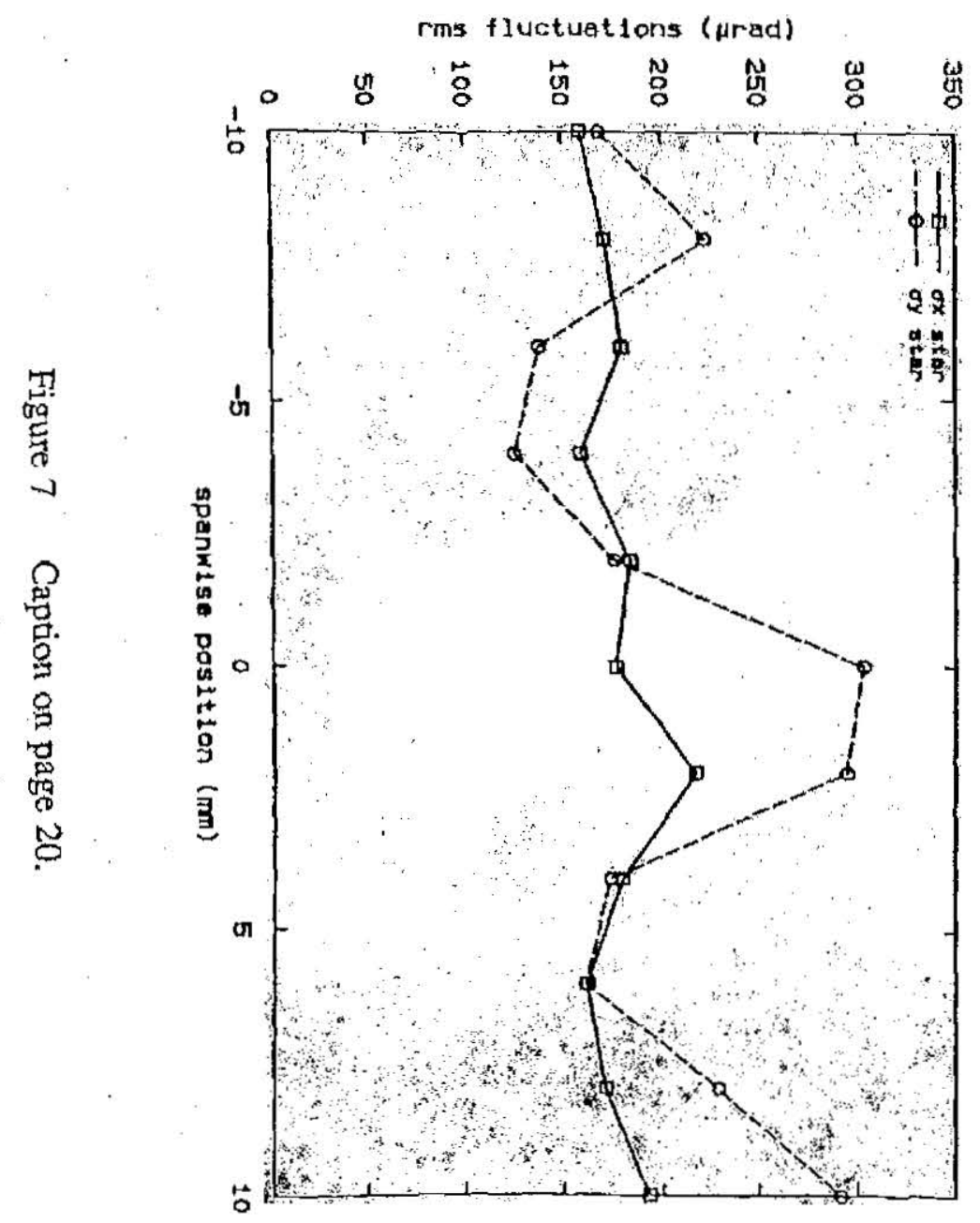

$\Xi$

ms fluctuations (urad)

N

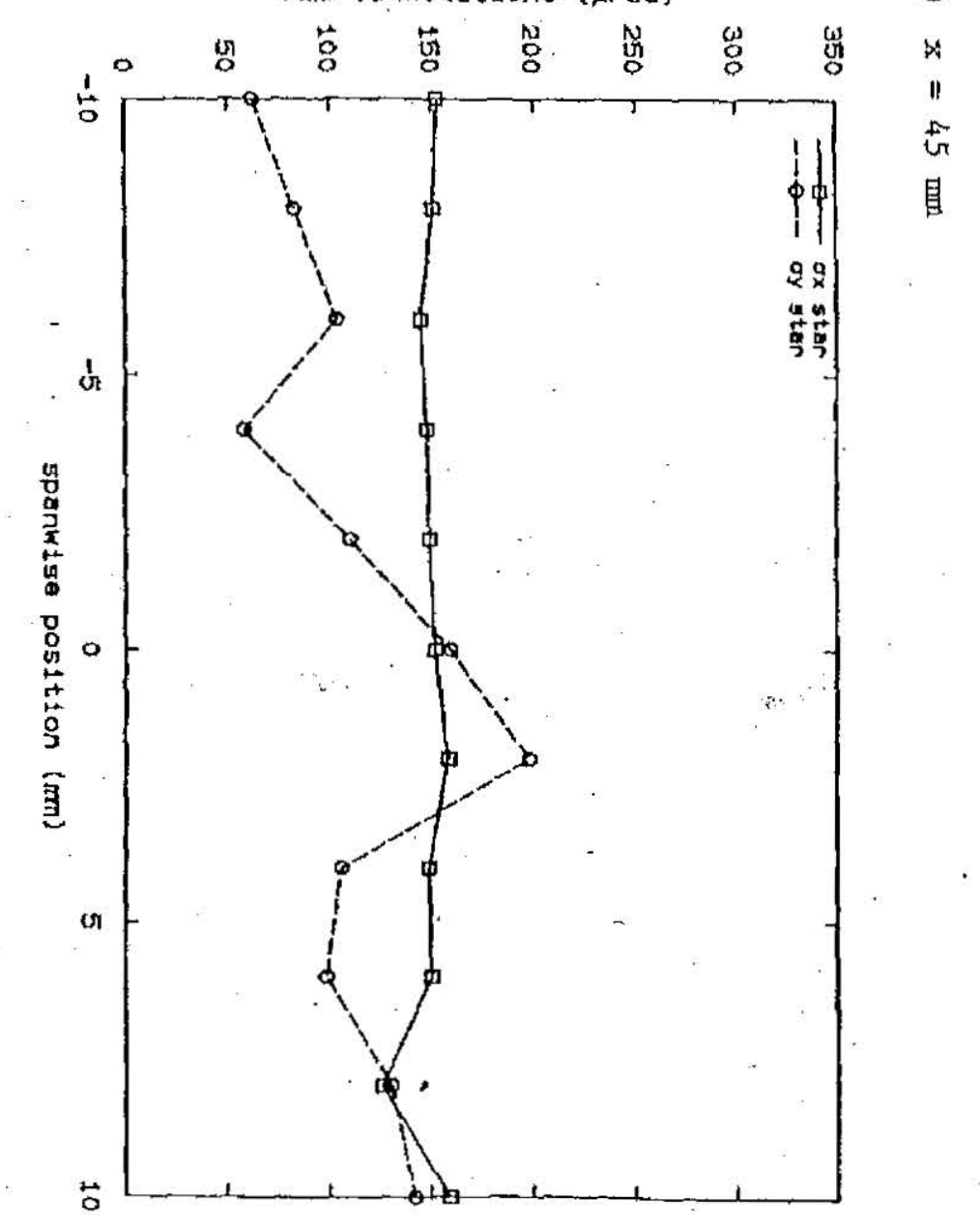


c) $x=113 \mathrm{~mm}$

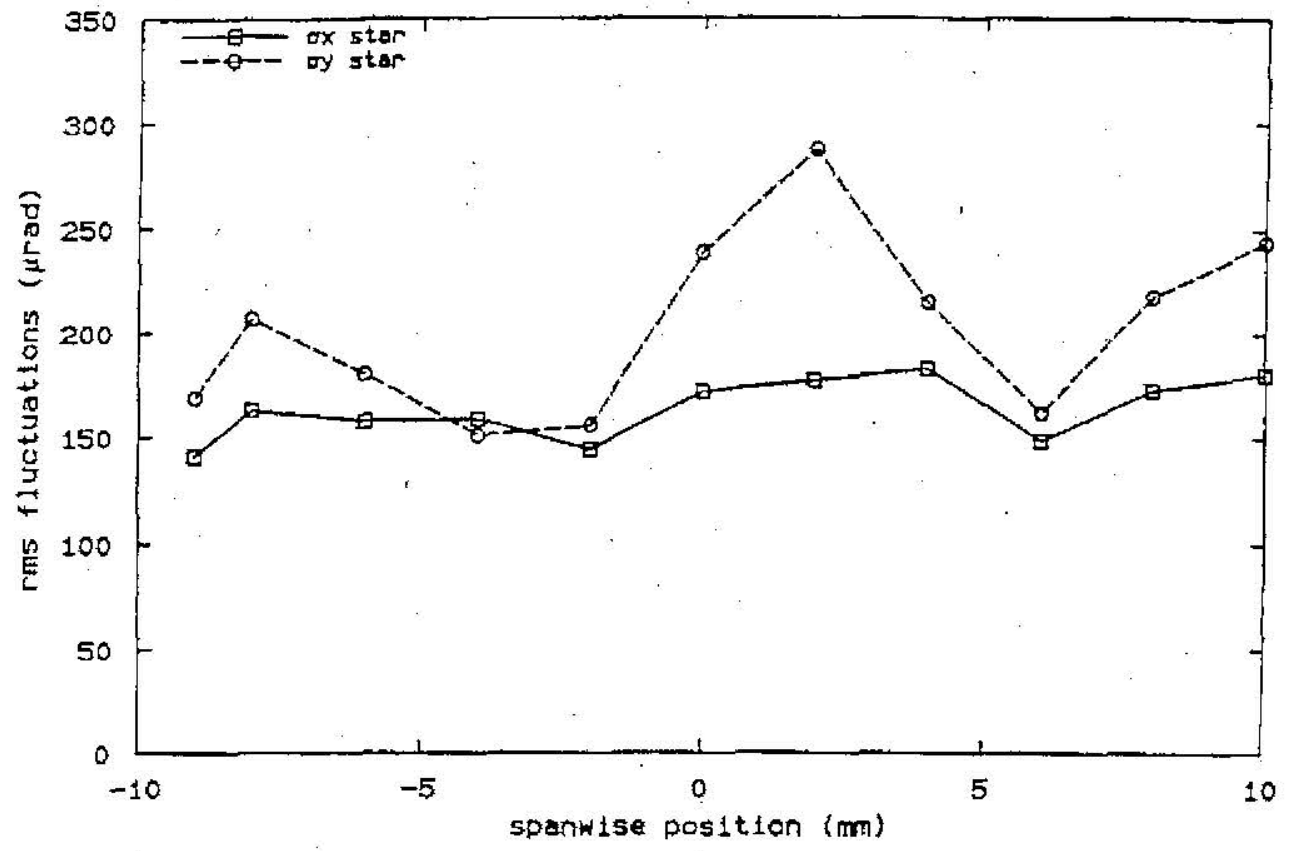

Figure 7 Spanwise distributions of $m$ sms fiuctuation, $\sigma_{\theta_{x}^{*}}$ and $\sigma_{\theta_{y}^{*}}$ vs. $y$ $\left(\mathrm{p}=2 \mathrm{bar} ; U_{1}=10.0 \mathrm{~m} / \mathrm{s}\right) ; U_{2}=3.8 \mathrm{~m} / \mathrm{s}$.

(a) $x=45 \mathrm{~mm}$; (b) $x=88 \mathrm{~mm}$; (c) $x=113 \mathrm{~mm}$. 
$\vdots$

a) $y=0$

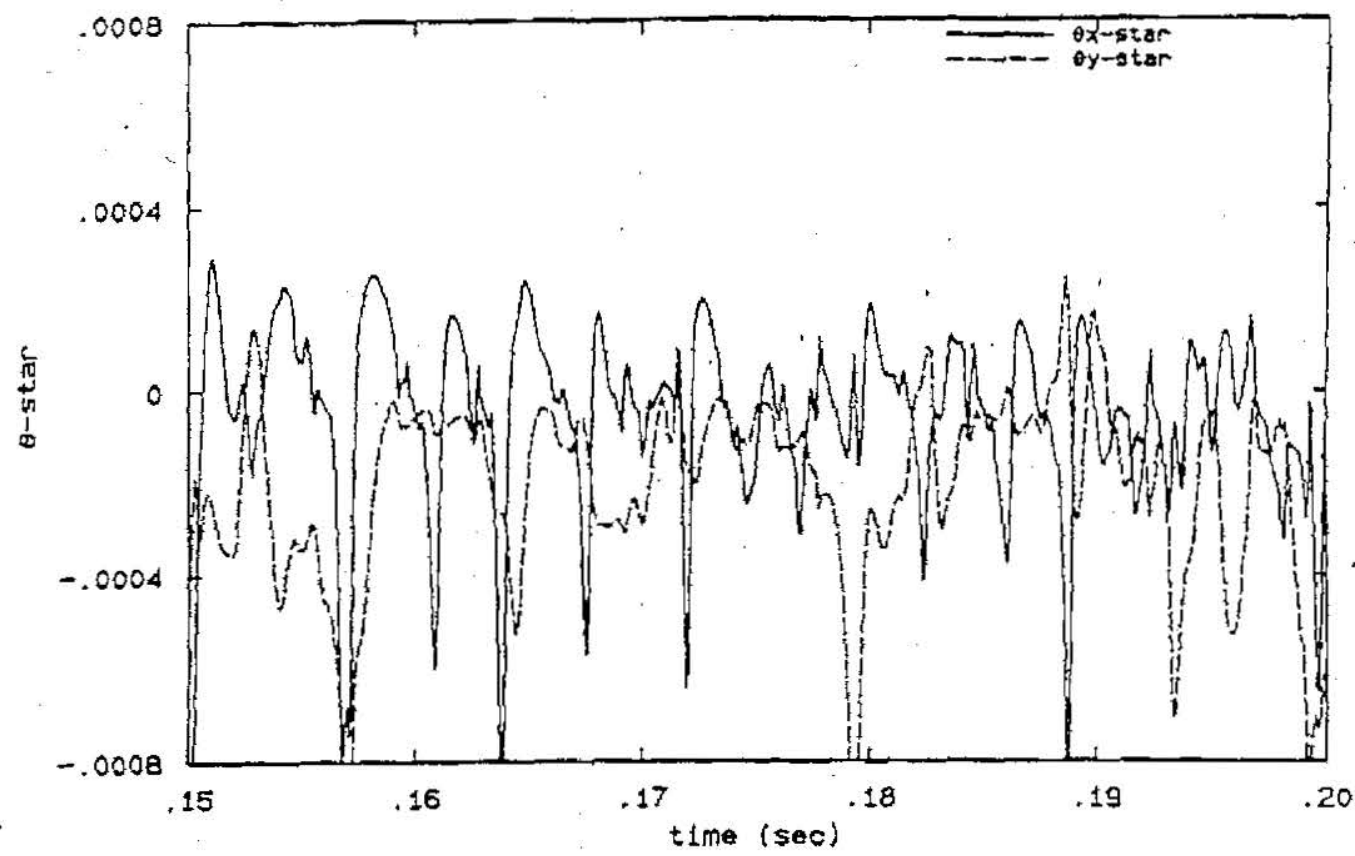

b) $y=2 m$

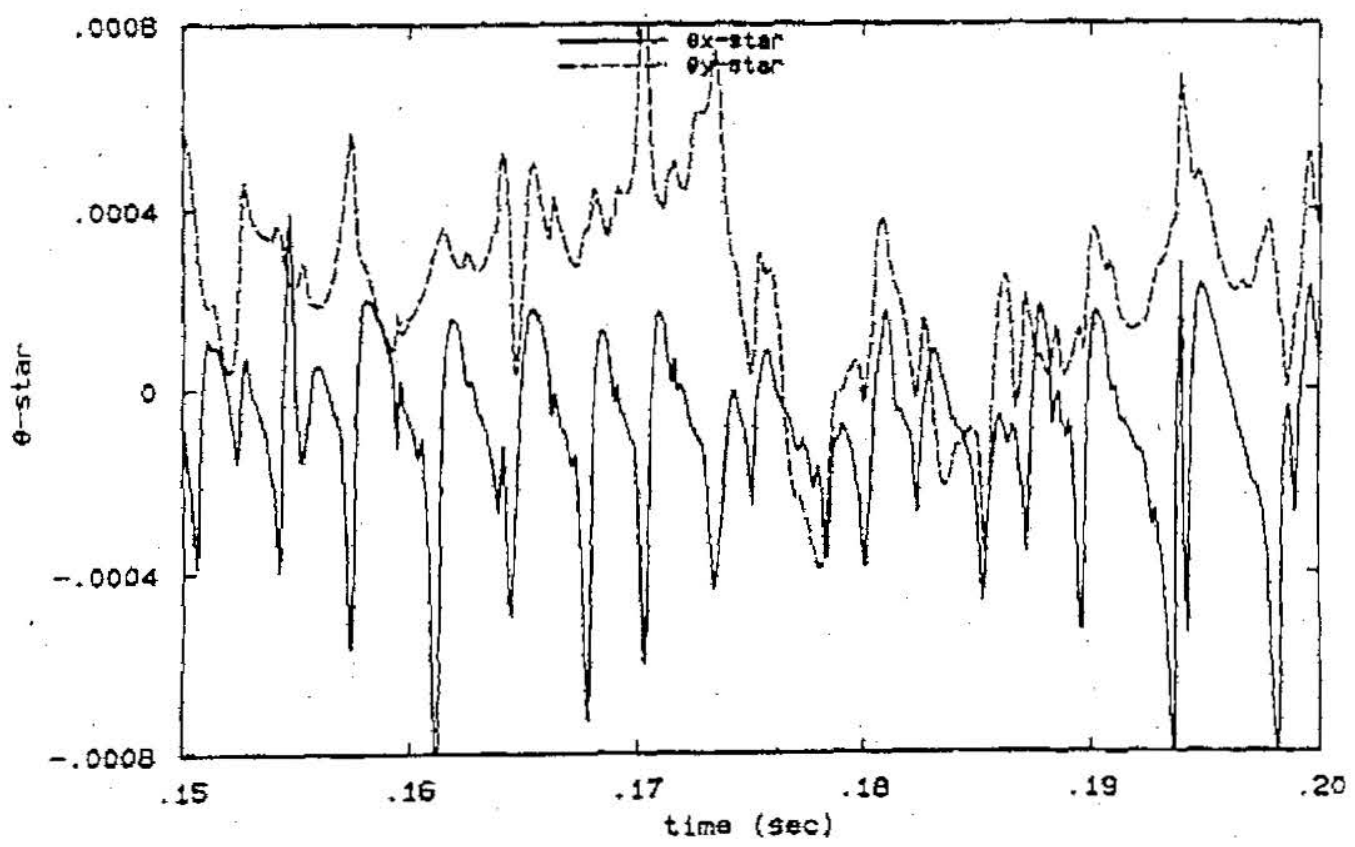

Figure 8 Caption on page 23. 
c) $y=4 \mathrm{~mm}$

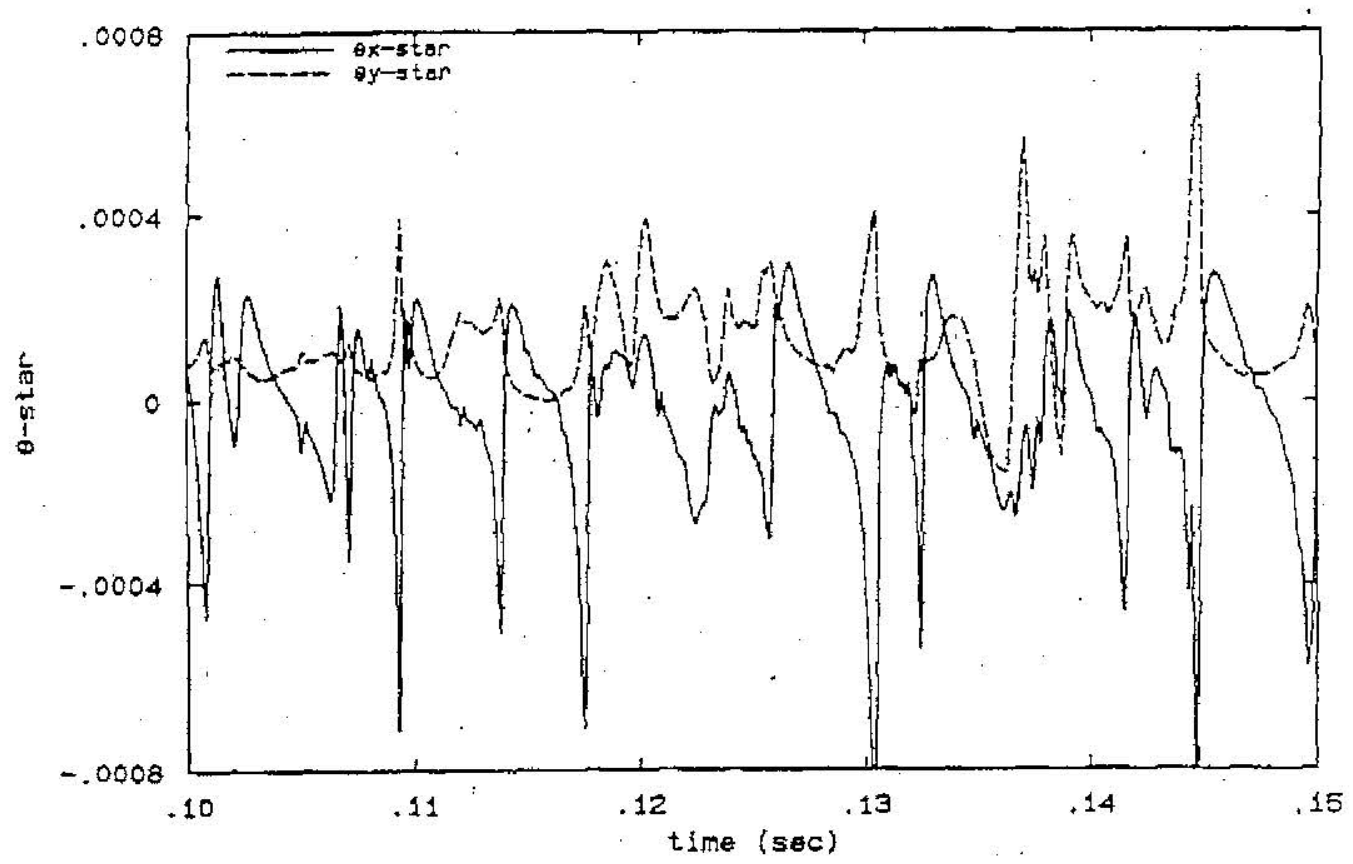

d) $y=6 \mathrm{nen}$

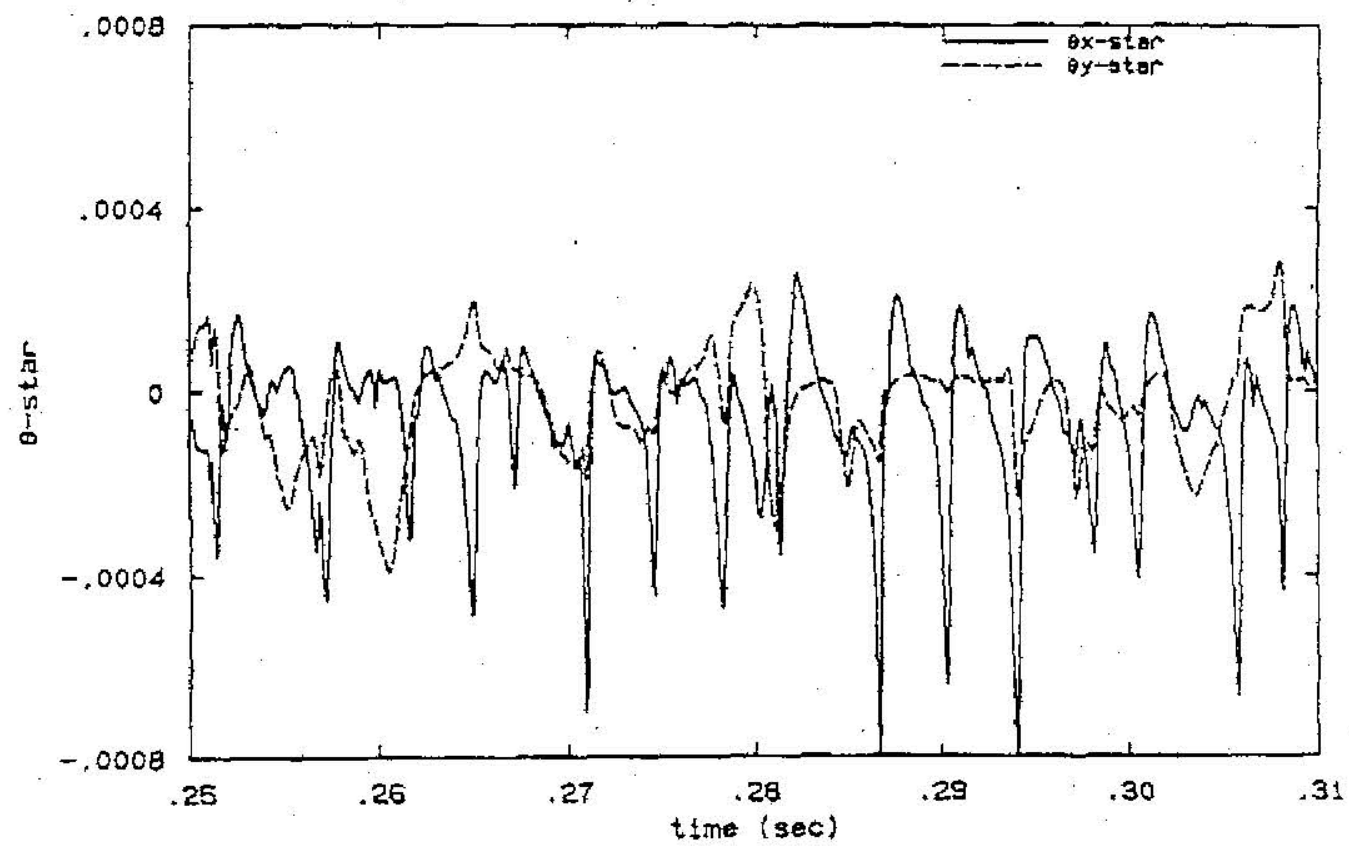

Figure 8 Caption on page 23. 
e) $y=8 m$

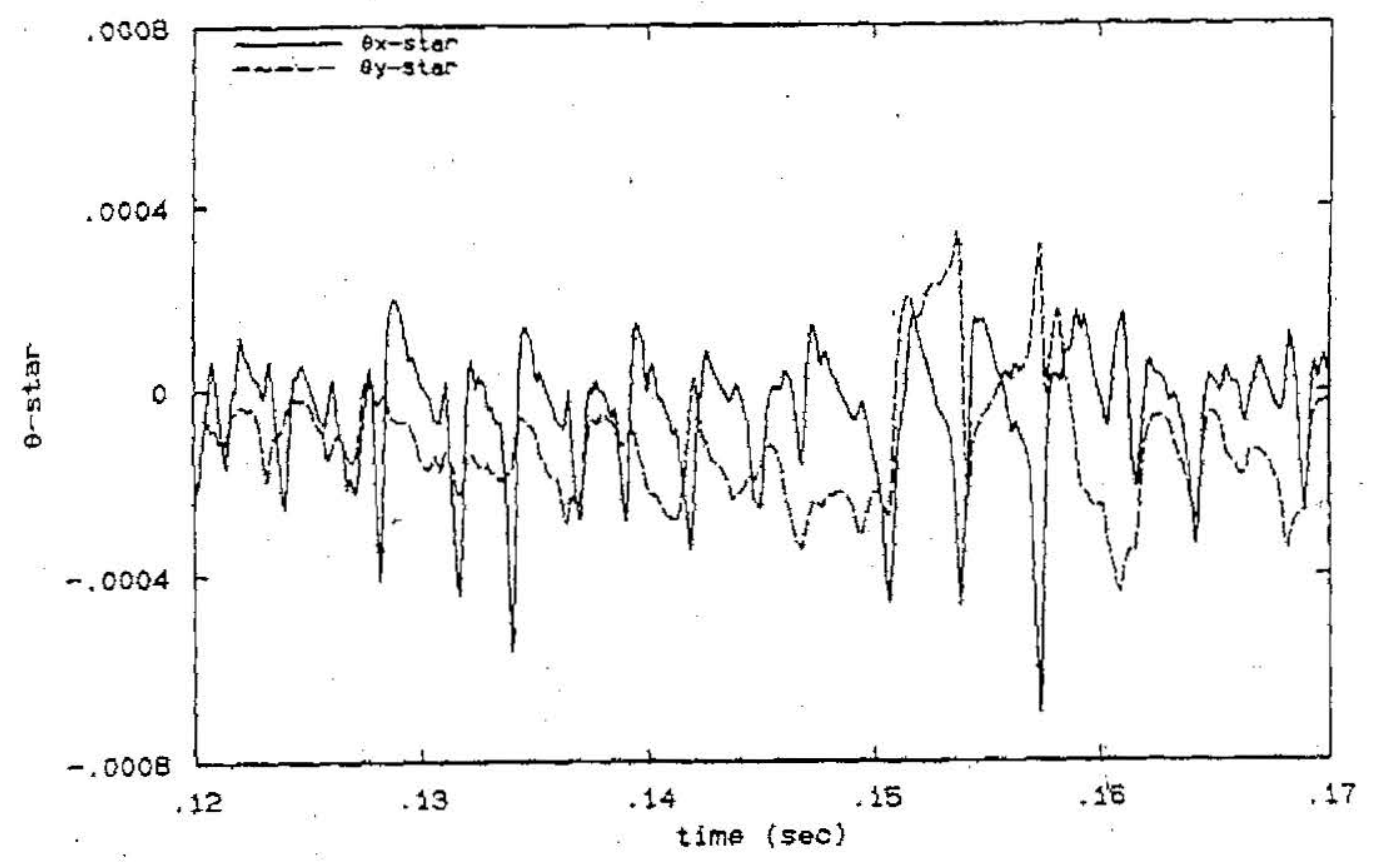

f) $y=10 \mathrm{~mm}$

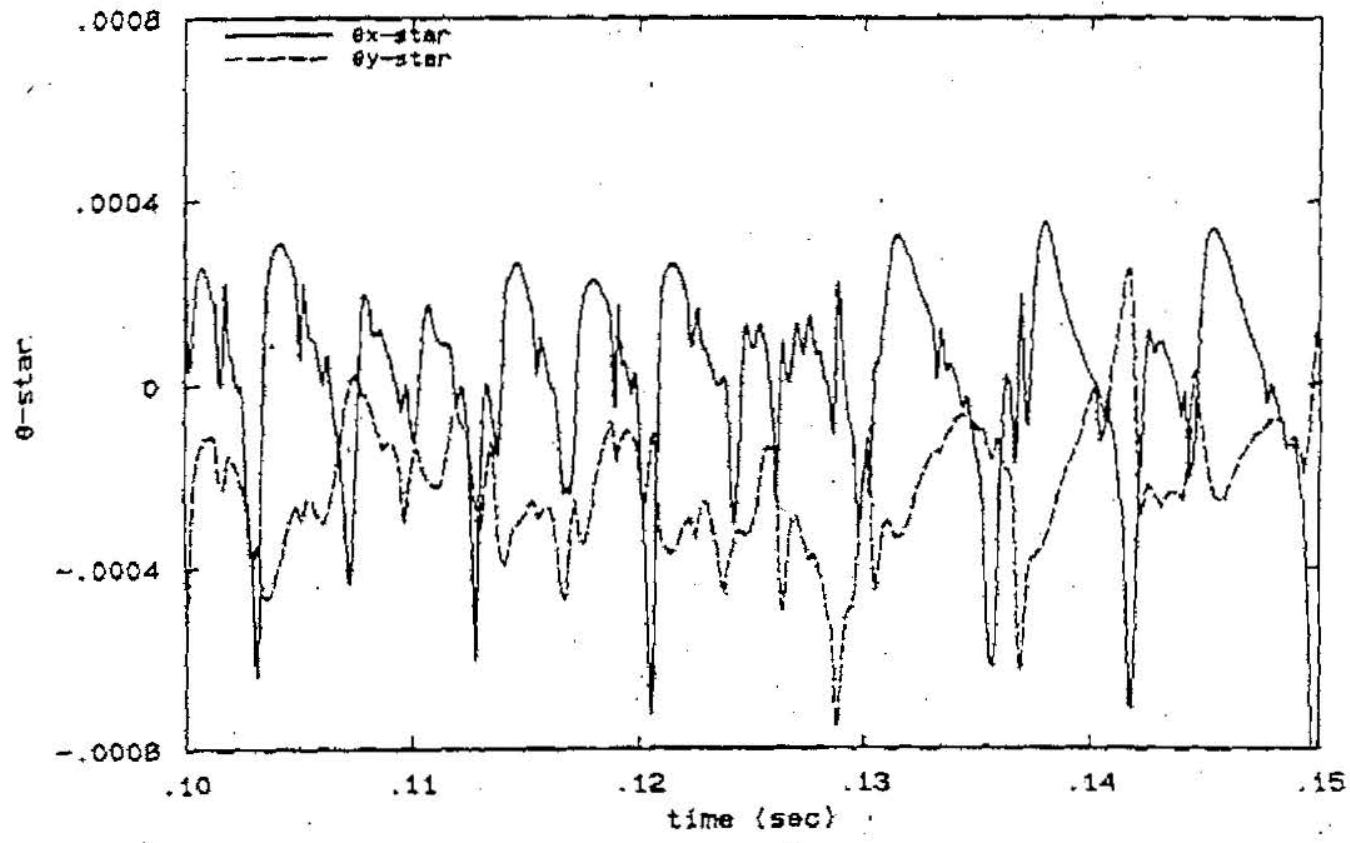

Figure 8 Deflection-angle time histories at different spanwise positions, $y$. $\left(\mathrm{p}=2 \mathrm{bar} ; U_{1}=10.0 \mathrm{~m} / \mathrm{s} ; U_{2}=3.8 \mathrm{~m} / \mathrm{s} ; x=45 \mathrm{~mm}\right) ;$

(a) $y=0 \mathrm{~mm}$; (b) $y=2 \mathrm{~mm}$; (c) $y=4 \mathrm{~mm}$;

(d) $y=6 \mathrm{~mm}$; (e) $y=8 \mathrm{~mm}$; (f) $y=10 \mathrm{~mm}$. 
a)

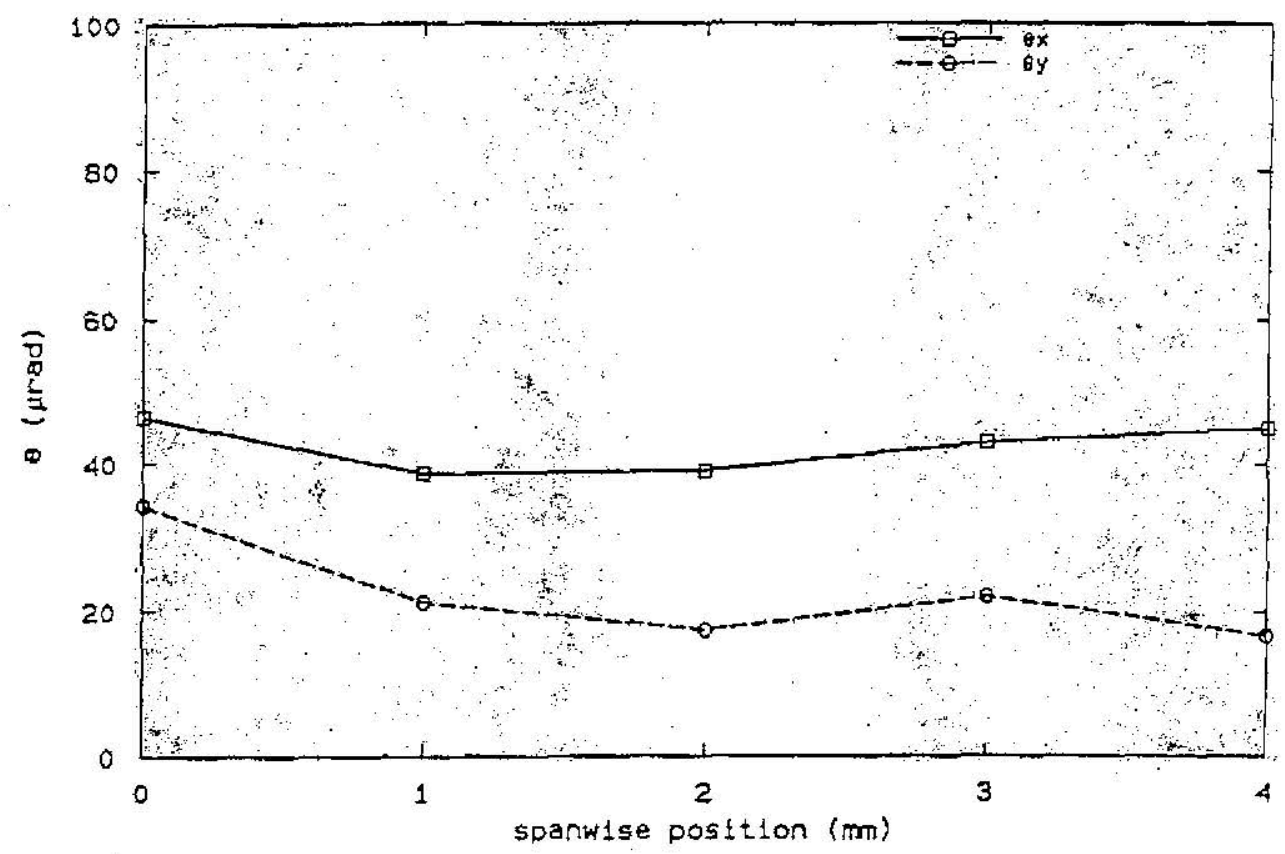

b)

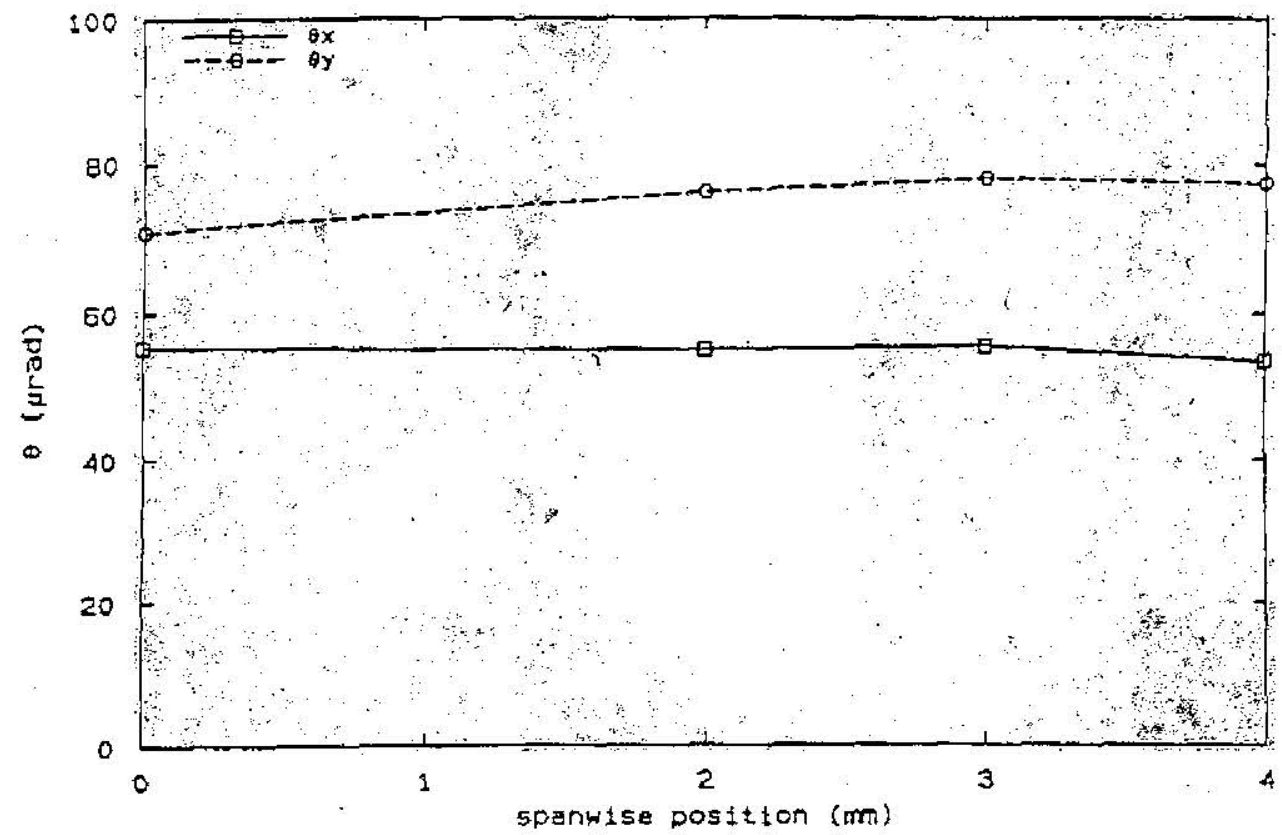

Figure 9 Spanwise distributions of ms fluctuation, $\sigma_{\theta_{x}^{*}}$ and $\sigma_{\theta_{j}^{*}} v s . y$, for $N_{2} / H e-A r$ mixing layer, $\left(\mathrm{p}=4\right.$ bar, $U_{1}=5.0 \mathrm{~m} / \mathrm{s} ; U_{2}=1.9 \mathrm{~m} / \mathrm{s} ; \delta_{1}^{* *}=0.083 \mathrm{~mm}$ )
(a) $x / \delta_{1}^{* *}=250$
(b) $x / \delta_{1}^{* *}=1355$ 
(a)

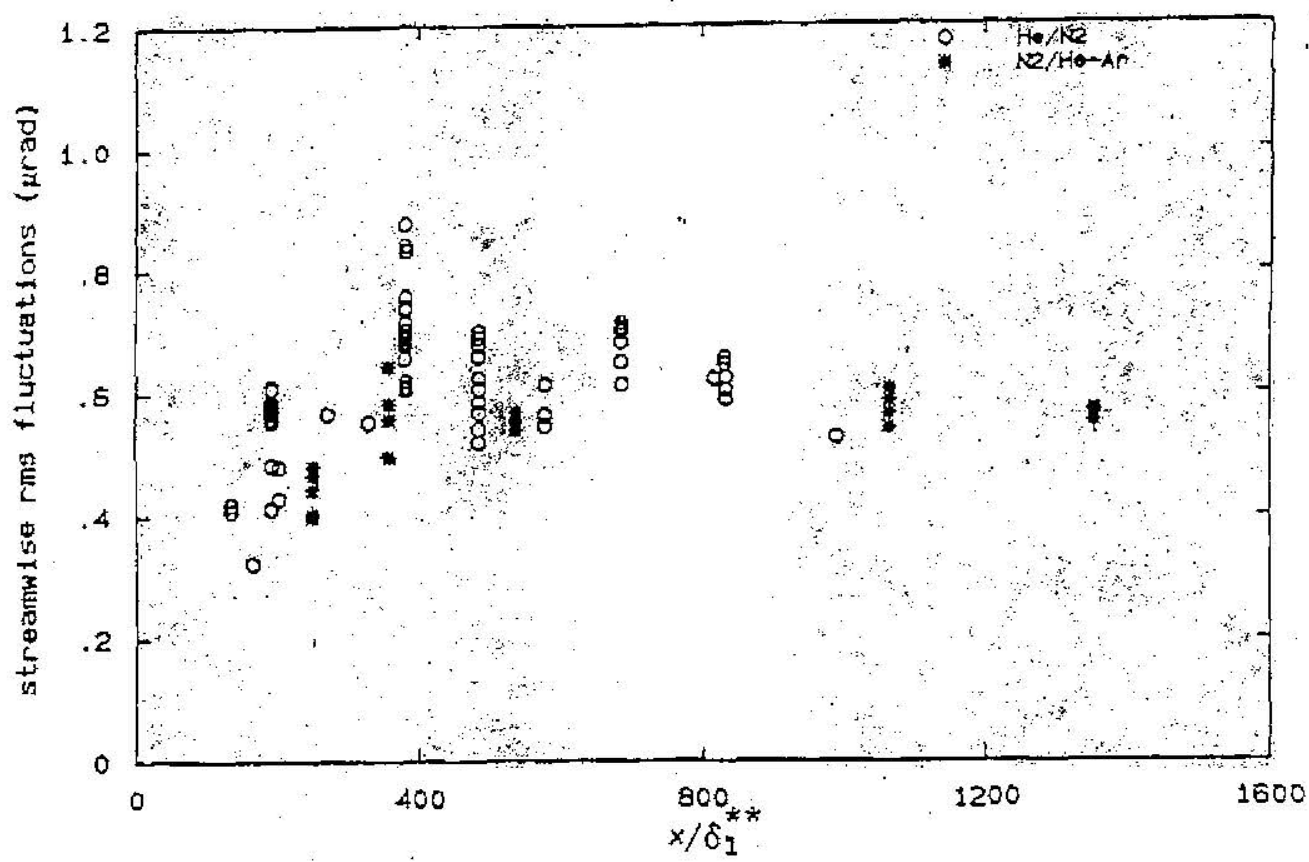

(b)

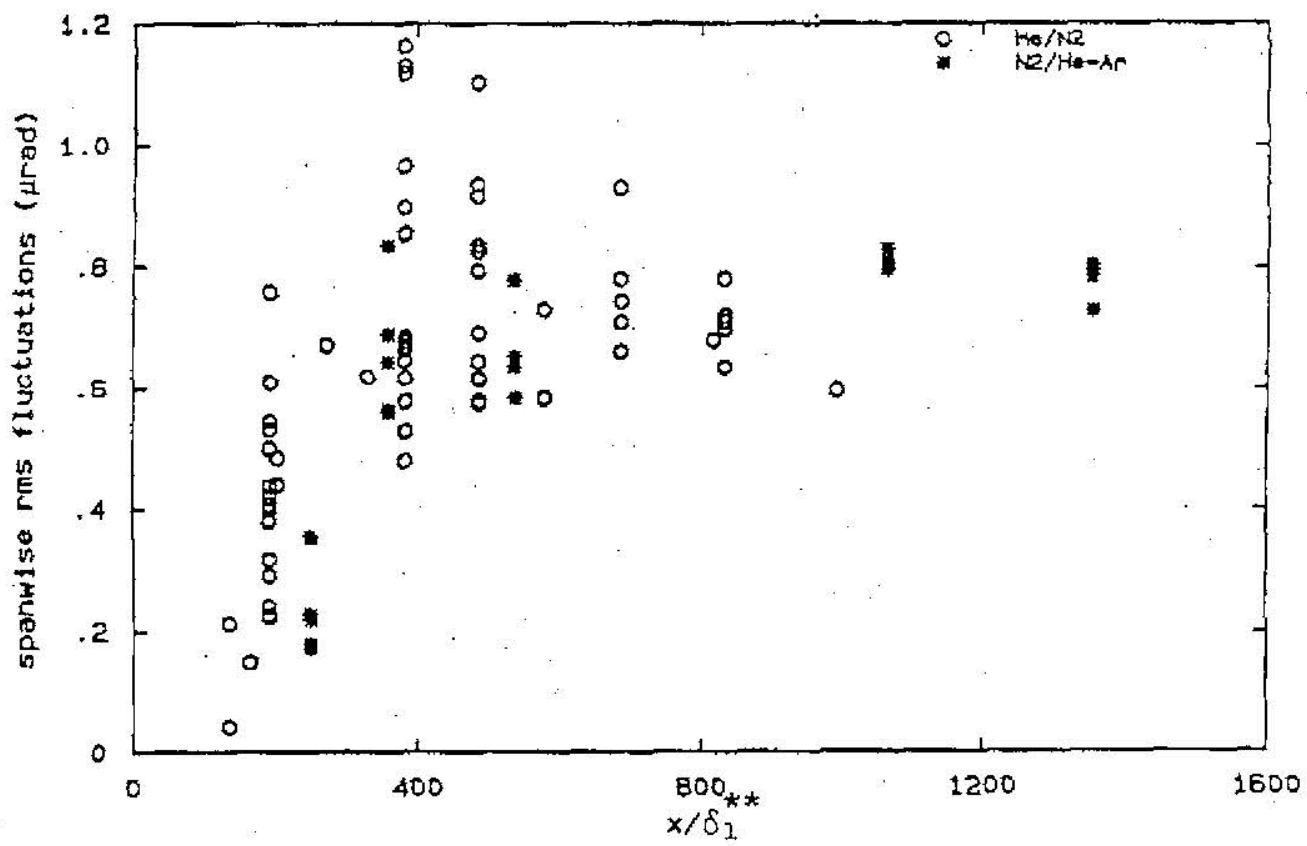

Figure 10 Fully scaled rms fluctuation vs $x / \delta_{1}^{* *}$

(a) Streamwise rms fluctuation, $\sigma_{\theta_{x s c}}$.

(b) Spanwise rms fluctuation, $\sigma_{\theta_{y} s c}$. 


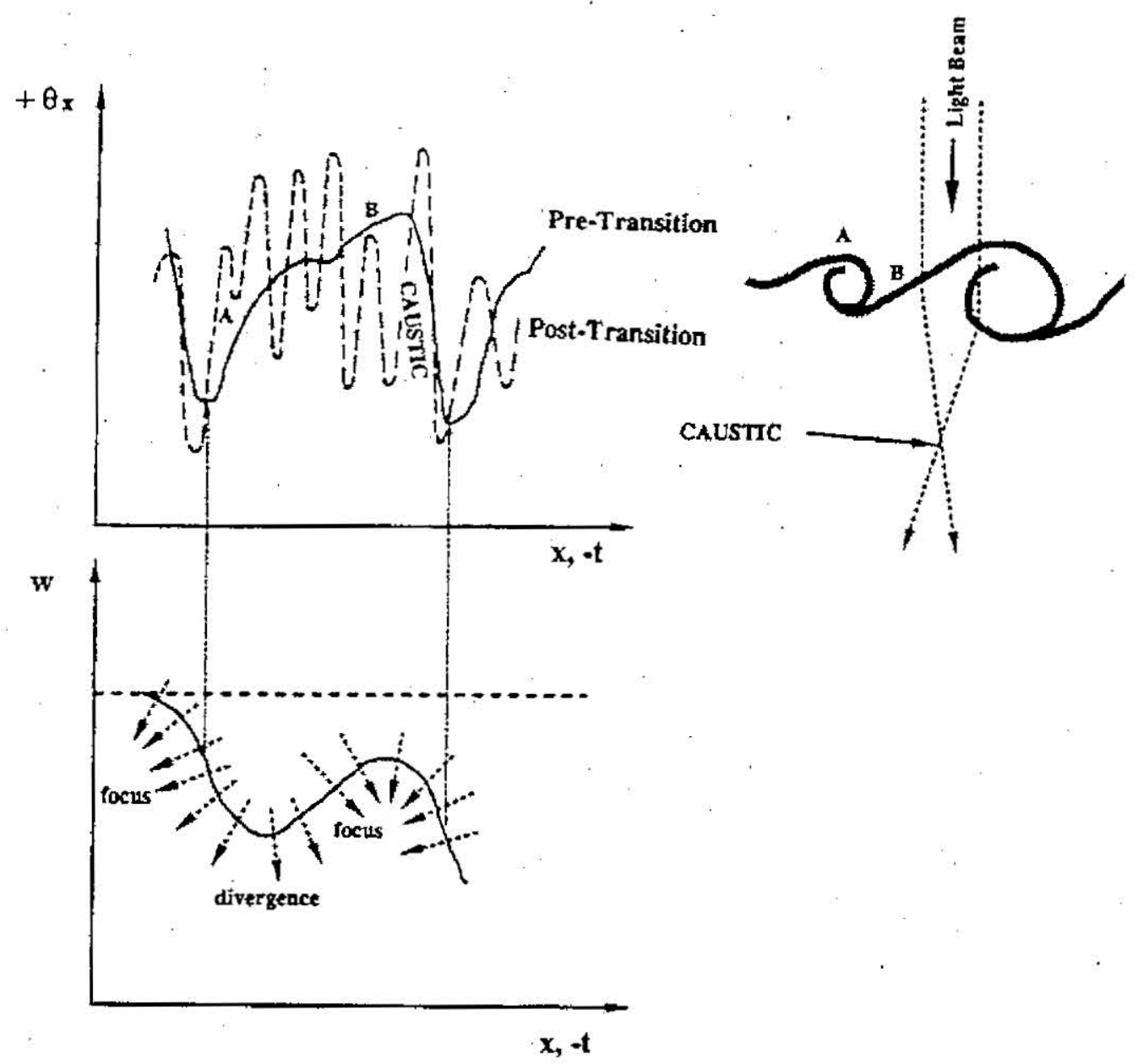

Figure 11 Model of spanwise coherent structure effect on a thin light beam $(\Delta \beta>0)$. 


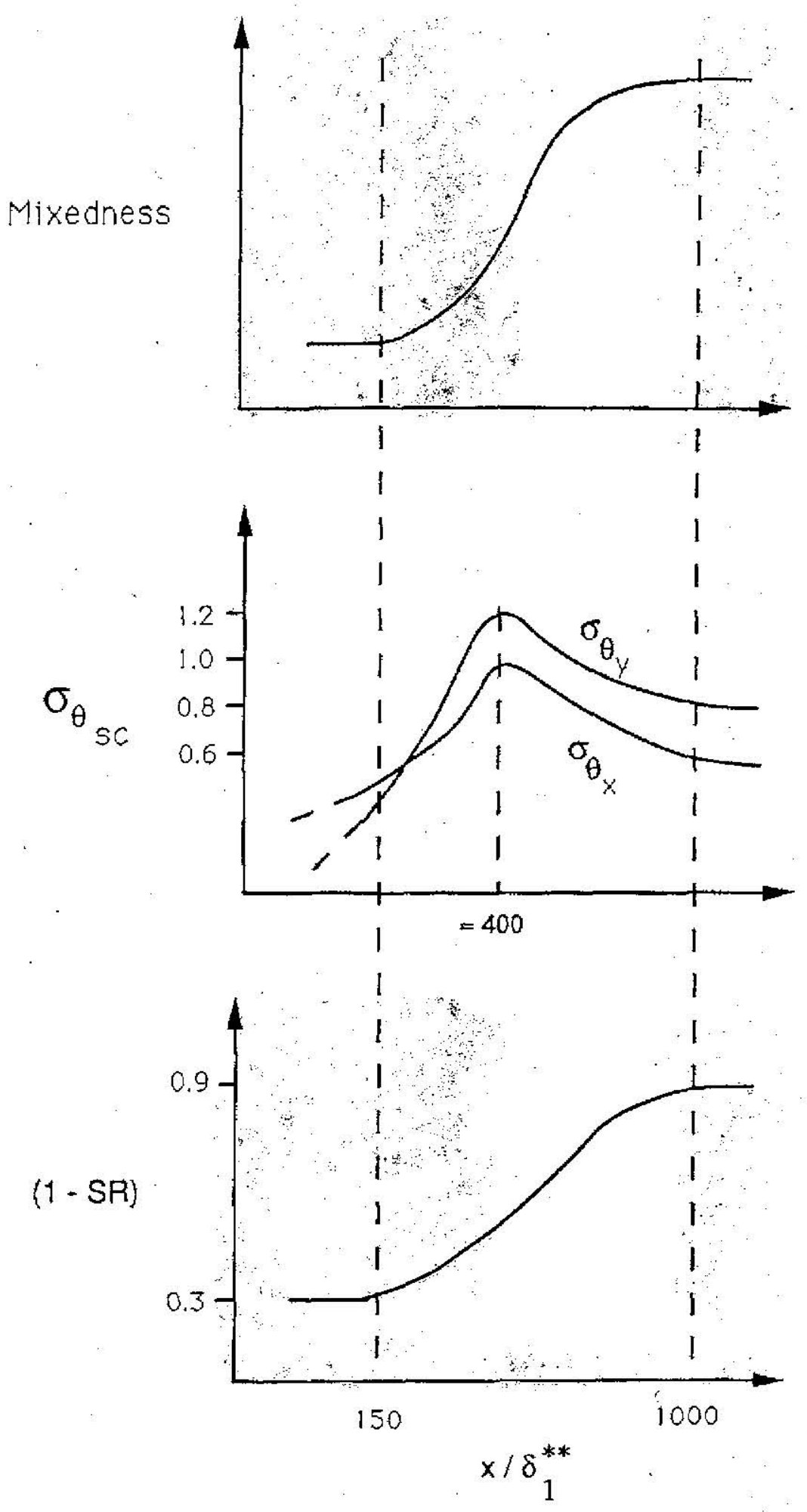

Figure 12 Relation of mixing transition to distributions of ms fluctuation $\left(\sigma_{B_{s S C}}\right.$ and $\left.\sigma_{\theta_{y s C}}\right)$ and optical abberation (1-SR). 